\title{
Mesoporous composite nanoparticles for dual- modality ultrasound/magnetic resonance imaging and synergistic chemo-/thermotherapy against deep tumors
}

\author{
This article was published in the following Dove Press journal: \\ International Journal of Nanomedicine \\ 5 October 2017 \\ Number of times this article has been viewed
}

\author{
Nan Zhang' \\ Ronghui Wang ${ }^{2}$ \\ Junnian $\mathrm{Hao}^{\prime}$ \\ Yang Yang \\ Hongmi Zou ${ }^{3}$ \\ Zhigang Wang' \\ 'Chongqing Key Laboratory of \\ Ultrasound Molecular Imaging, Second \\ Affiliated Hospital of Chongqing \\ Medical University, Chongqing Medical \\ University, Chongqing, ${ }^{2}$ Department \\ of Ultrasound, Ruijin Hospital, \\ Shanghai Jiao Tong University School \\ of Medicine, Shanghai, ${ }^{3}$ Department \\ of Ophthalmology, Second Affiliated \\ Hospital of Chongqing Medical \\ University, Chongqing, People's \\ Republic of China
}

\begin{abstract}
High-intensity focused ultrasound (HIFU) is a promising and noninvasive treatment for solid tumors, which has been explored for potential clinical applications. However, the clinical applications of HIFU for large and deep tumors such as hepatocellular carcinoma (HCC) are severely limited by unsatisfactory imaging guidance, long therapeutic times, and damage to normal tissue around the tumor due to the high power applied. In this study, we developed doxorubicin/perfluorohexane-encapsulated hollow mesoporous Prussian blue nanoparticles (HMPBs-DOX/PFH) as theranostic agents, which can effectively guide HIFU therapy and enhance its therapeutic effects in combination with chemotherapy, by decreasing the cavitation threshold. We investigated the effects of this agent on ultrasound and magnetic resonance imaging in vitro and in vivo. In addition, we showed a highly efficient HIFU therapeutic effect against HCC tumors, as well as controlled drug release, owing to the phase-transitional performance of the PFH. We therefore conclude that HMPB-DOX/PFH is a safe and efficient nanoplatform, which holds significant promise for cancer theranostics against deep tumors in clinical settings.
\end{abstract}

Keywords: high-intensity focused ultrasound, HIFU, hollow mesoporous Prussian blue nanoplatforms, hepatocellular carcinoma, dual-modality imaging, synergistic chemo-/thermotherapy, theranostics

\section{Introduction}

Hepatocellular carcinoma (HCC) is the third most prevalent life-threatening disease in the world and is a highly unpredictable condition involving heterogeneous pathologies that cause tremendous challenges for early diagnosis and effective treatment. ${ }^{1-3}$ According to the Barcelona's Clinic Liver Cancer staging system for HCC, only stage A HCC is amenable to resection, liver transplantation, or local ablation for tumor therapy. ${ }^{4-7}$ The chances of successful treatment are therefore reduced in advanced HCC, as well as when the tumor is located at the hepatic hilar, or when there are limited suitable liver donors available. ${ }^{8,9}$ High-intensity focused ultrasound (HIFU) may be an alternative therapeutic method to chemotherapy for these patients. ${ }^{10-13}$ Compared with other ablation treatments, ${ }^{14-16}$ HIFU is a noninvasive and precise treatment, wherein the ultrasound source is focused on a targeted area with millimeter precision to ablate the tumor inside the body. ${ }^{17}$ Ultrasound combined with drug-loaded agents can also enhance cavitation effects, as well as avoiding tumor recurrence caused by heterogeneous ablation. ${ }^{18,19}$
Correspondence: Zhigang Wang Second Affiliated Hospital of Chongqing Medical University \& Chongqing Key Laboratory of Ultrasound Molecular Imaging, 74 Linjiang Road, Chongqing 400016 , People's Republic of China $\mathrm{Tel} / \mathrm{fax}+862363809074$

Emailwzg62942443@163.com 
Various synergistic agents (SAs), including organic microbubbles, organic nanoemulsions, and inorganic nanoparticles, have been fabricated to promote the HIFU therapeutic effect. ${ }^{20-23}$ These SAs serve as effective cavitation nuclei, significantly reducing the cavitation threshold and changing the acoustic environment, thus enhancing the energy deposition and the efficacy and safety of HIFU therapy. ${ }^{24}$ Moreover, the SAs are not only used for enhancing therapeutic efficacy but also applied as contrast agents (CAs) to image the tumor region, which may help to achieve more precise imaging guidance and assessment of therapeutic outcome. Sun et al ${ }^{22}$ described superparamagnetic poly(lacticco-glycolic acid) (PLGA) microcapsules $\left(\mathrm{Fe}_{3} \mathrm{O}_{4} / \mathrm{PLGA}\right)$ for dual-modality biological imaging and enhancement of HIFU therapeutic efficiency for breast cancer. Yao et $\mathrm{a}^{25}$ showed that integration of $\mathrm{Bi}_{2} \mathrm{~S}_{3}$ and PLGA nanocapsules can enhance ultrasound imaging-guided HIFU/radiotherapy synergistic therapy. Nevertheless, these organic/inorganic hybrid nanocapsules typically involve a high cost of fabrication and complex synthetic steps, which hinder their further clinical applications. ${ }^{26,27}$ Therefore, there is an urgent need to develop a single-component, cost-effective, and biocompatible/stable nanoplatform to improve multimodality imaging and HIFU therapeutic efficacy for HCC.

Prussian blue (PB), a mixed-valence iron hexacyanoferrate, has attracted much attention in recent years and has been approved by the US Food and Drug Administration (FDA) for the treatment of radiation exposure. It exhibits the properties of intrinsic paramagnetism, which negatively enhances magnetic resonance imaging (MRI) T2-weighted contrast. In particular, owing to the tunable morphology of $\mathrm{PB}$ in the forms of solid, shell, or hollow nanostructures, ${ }^{28-31}$ it allows $\mathrm{PB}$, as a versatile drug carrier, to deliver drugs into the tumor site with high drug-loading efficiency. ${ }^{32}$ Our previous research has demonstrated that hollow mesoporous PB nanoparticles (HMPBs) can be used to encapsulate chemotherapeutic drugs and perfluorohexane (PFH), thereby enhancing HIFU therapy in subcutaneous xenograft tumors.

In this study, we prepared doxorubicin/PFH-encapsulated HMPBs (HMPBs-DOX/PFH) as theranostic agents for HCC ablation. Based on our previous study, we further investigated the potential of our HMPBs-DOX/PFHs to improve MRI and synergistic HIFU ablation in deep tumors. In contrast to the previous study, we focused on MRI instead of photoacoustic imaging, since MRI has a better penetration than photoacoustic imaging, and it is therefore more appropriate for guiding deep tumor ablation. We also investigated the effects of HMPB-DOX/PFH therapy on tumor recurrence, compared with heterogeneous ablation (Scheme 1).

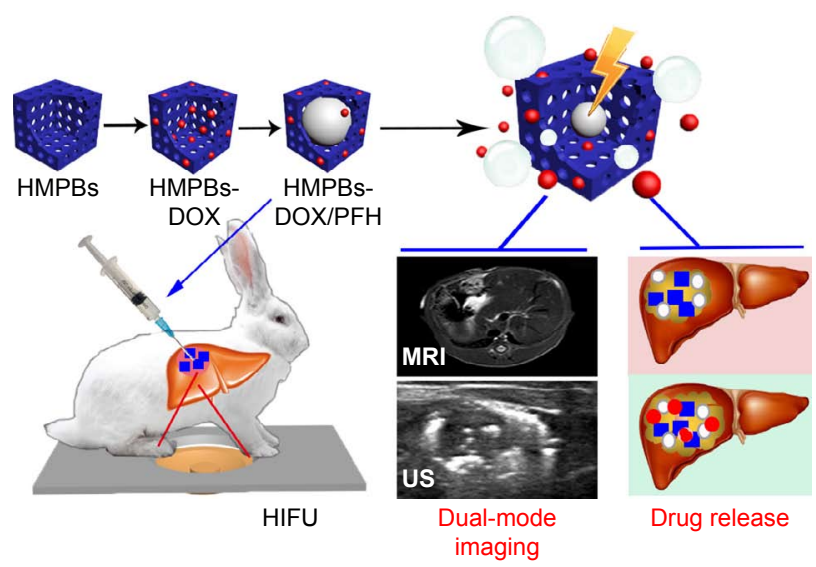

Scheme I Schematic of the synthetic procedure for HMPBs-DOX/PFH and how this SA intensifies US and MRI, as well as improving the treatment efficacy of HIFU ablation for tumor and residual tumor cells.

Abbreviations: DOX, doxorubicin; HIFU, high-intensity focused ultrasound; HMPBs, hollow mesoporous Prussian blue nanoparticles; MRI, magnetic resonance imaging; PFH, perfluorohexane; SA, synergistic agent; US, ultrasound; HMPBs-DOXI $\mathrm{PFH}, \mathrm{DOX} / \mathrm{PFH}$-encapsulated HMPBs.

\section{Materials and methods Synthesis of HMPBs}

Polyvinylpyrrolidone (PVP) K30, potassium ferricyanide $\left(\mathrm{K}_{3}\left[\mathrm{Fe}(\mathrm{CN})_{6}\right]\right)$, hydrochloric acid $(\mathrm{HCl}, 36.0 \%-38.0 \%)$, and PFH (Sigma-Aldrich Co., St Louis, MO, USA) were purchased from Sinopharm Chemical Reagent Co., Ltd (Shanghai, China). $\operatorname{PVP}(3.0 \mathrm{~g})$ and $\mathrm{K}_{3}\left[\mathrm{Fe}(\mathrm{CN})_{6}\right](132 \mathrm{mg})$ were mixed with $\mathrm{HCl}$ solution $(0.01 \mathrm{M}, 40 \mathrm{~mL})$ and stirred magnetically until a clear solution is obtained. The mixed solution was placed in an electric oven at $80^{\circ} \mathrm{C}$ for $24 \mathrm{~h}$ to obtain the mesoporous PB nanoparticles (MPBs). The MPBs were washed sequentially with distilled water $(3 \times 1 \mathrm{~mL})$ three times to remove the unreacted chemicals. PVP $(5 \mathrm{mg} / \mathrm{mL})$ was added to the solution of MPBs $(1 \mathrm{mg} / \mathrm{mL})$ in a Teflon vessel and kept for $3 \mathrm{~h}$ under magnetic stirring. The solution was then transferred to an autoclave and kept at $140^{\circ} \mathrm{C}$ for 4 days. Finally, the HMPBs were collected and washed at least three times with distilled water.

\section{Synthesis of PFH and drug-loaded HMPBs (HMPBs-DOX/PFH)}

HMPBs were added to appropriate concentrations of DOX in phosphate-buffered saline (PBS) and reacted for $24 \mathrm{~h}$ at room temperature in the dark. The product (HMPBs-DOX) was collected by centrifugation and washed with PBS until the supernatant was clear, with no color being visibly detectable. Then, the HMPBs-DOX were dried under vacuum at room temperature. The vacuum-dried samples (HMPBsDOX, $25 \mathrm{mg}$ ) were added to $75 \mu \mathrm{L}$ of temperature-sensitive $\mathrm{PFH}$ liquid. The samples were then oscillated in ice water for 2 min to facilitate the loading of the PFH into the hollow core. 
Finally, the HMPBs-DOX/PFH were mixed with $5 \mathrm{~mL}$ of deionized water under slight magnetic stirring.

\section{Characterization of HMPBs-DOX/PFH}

The morphologies and element mappings of the HMPBs were observed by a transmission electron microscope (TEM, JSM-2100F; JEOL, Tokyo, Japan). An energy-dispersed spectrometer (EDS, JSM-2100F; JEOL) was used to determine the element ratios of HMPBs. The particle sizes and zeta potentials of the nanoparticles were determined using a laser particle size analyzer system (Zetasizer Nano ZS90; Malvern Instruments, Malvern, UK). The drug-loading capacity was then calculated by the following equation using high-performance liquid chromatography (HPLC) LC-2010A HT, Agilent 1100 (Shimadzu, Kyoto, Japan).

\section{Loading capacity $=\left(\frac{\text { Total DOX }- \text { Unbound DOX }}{\text { Total HMPBs }}\right)$.}

For the safety of HMPBs-DOX/PFH in vivo experiment, the stability of these hybrid mesoporous nanoparticles was tested by measuring the average diameter at $37^{\circ} \mathrm{C}$ at different time points (Particle Sizing Systems, Inc., Santa Barbara, CA, USA). Then, HMPBs-DOX/PFH were heated with a heating panel and visualized using an inverted fluorescence microscope (Olympus Corporation, Tokyo, Japan) to determine whether PFH had been successfully encapsulated into HMPBs-DOX. Approximately $50 \mu \mathrm{L}$ of diluted HMPBsDOX/PFH was dropped onto the microscope slide, which was put on the thermostatic plate. After heating the thermostatic plate (from room temperature to $70^{\circ} \mathrm{C}$ ), the sample was observed under an optical microscope. HMPBs-DOX were used as the control using the same methods.

\section{In vitro release of DOX from the HMPBs-DOX/PFH by double stimuli}

In vitro release profiles for HMPBs-DOX/PFH were investigated at two different $\mathrm{pH}$ values ( $\mathrm{pH} 5.0$ and 7.4). The nanoplatform solutions were placed in two dialysis bags (DOX $1 \mathrm{mg} / \mathrm{mL}$ in $200 \mathrm{~mL}$ PBS) and incubated at each $\mathrm{pH}$ for $48 \mathrm{~h}$. The release of DOX at each $\mathrm{pH}$ value was determined using HPLC at specific time intervals. The percentage of the released DOX was calculated as $\left(\mathrm{I}_{\mathrm{t}}-\mathrm{I}_{0}\right) /\left(\mathrm{I}_{100}-\mathrm{I}_{0}\right)$ $\times 100 \%$, in which $I_{t}$ is the concentration at time point $t, I_{0}$ is the concentration at the start of the incubation time, and $\mathrm{I}_{100}$ is the concentration after the addition of $10 \mathrm{~mL}$ of $\mathrm{HCI}$ solution. To further evaluate the release capability of these drug carriers after HIFU exposure, the HMPBs-DOX/PFH were observed using the same method as described earlier, and the samples at the two different $\mathrm{pH}$ values were exposed to HIFU at $12 \mathrm{~h}$ with a power density of $120 \mathrm{~W}$ for $5 \mathrm{~s}$. Then, we detected the release capability of HMPBs-DOX and HMPBs-DOX/PFH under the same energy power at $\mathrm{pH} 5.0$ to evaluate whether the phase-transitional process could hinder the release of DOX from the HMPBs. The thermostability of the DOX was subsequently detected using HPLC.

\section{Cell culture}

The MDA-MB-231 human breast cancer cell line was obtained from the Cancer Epigenetics Lab (The Chinese University of Hong Kong, Hong Kong, China), which had been originally purchased from American Type Culture Collection (ATCC, Manassas, VA, USA). The cells were cultured in Dulbecco's Modified Eagle's Medium (DMEM), supplemented with $10 \%$ fetal bovine serum, $100 \mathrm{U} / \mathrm{mL}$ penicillin, and $100 \mathrm{U} / \mathrm{mL}$ streptomycin under a humidified atmosphere in $5 \% \mathrm{CO}_{2}$ at $37^{\circ} \mathrm{C}$. Cells in the exponential phase of growth were used for in vitro experiments.

\section{In vitro cell viability of HMPBs and antitumor efficacy}

MDA-MB-231 cells $\left(1 \times 10^{4}\right.$ cells/well $)$ were seeded in 96-well plates. After $24 \mathrm{~h}$ of incubation, they were added to different concentrations of HMPBs and incubated at $37^{\circ} \mathrm{C}$. Following incubation with HMPBs for $24 \mathrm{~h}$, the cells were washed twice with PBS, after which $10 \mu \mathrm{L}$ of 3-(4,5dimethylthiazol-2-yl)-2,5-diphenyltetrazolium bromide (MTT) solution $(5 \mathrm{mg} / \mathrm{mL})$ and $100 \mu \mathrm{L}$ fresh medium were added. After further incubation for $4 \mathrm{~h}$, the medium was replaced with dimethyl sulfoxide (DMSO; $150 \mu \mathrm{L}$ ). The absorbance was determined at $490 \mathrm{~nm}$ by a microplate reader (BioTek ELx800; BioTek Instruments, Inc., Winooski, VT, USA). The effect on cell viability of HMPBs at different concentrations was expressed as a percentage: $\left(\mathrm{OD}_{\text {sample }}-\mathrm{OD}_{\text {blank }}\right.$ ' $\left.\mathrm{OD}_{\text {control }}-\mathrm{OD}_{\text {blank }}\right) \times 100 \%$. For the assay, the experiments were performed in triplicate.

Before carrying out the cell apoptosis experiment, it was necessary to verify the phagocytosis of nanoplatforms by MDA-MB-231 cells. These breast cancer cells, at a density of $1 \times 10^{5}$ cells/well, were seeded in a six-well plate containing a coverslip in each well. After $24 \mathrm{~h}$ of culture, $1 \mathrm{~mL}$ of HMPBs$\mathrm{DOX} / \mathrm{PFH}(100 \mu \mathrm{g} / \mathrm{mL})$ was added to the cells, and they were incubated for $2 \mathrm{~h}$. MDA-MB-231 cells were washed with PBS three times, fixed with $4 \%$ paraformaldehyde for $15 \mathrm{~min}$, and stained with 2-(4-amidinophenyl)-6-indolecarbamidine dihydrochloride (DAPI) for visualization of the nuclei and 3,3'-dioctadecyloxacarbocyanine perchlorate (DiO) for visualization of the cell membrane. The stained coverslips 
were photographed using confocal laser scanning microscopy (CLSM; Nikon A1 confocal microscope system; Nikon Corporation, Tokyo, Japan). DOX, DAPI, and DiO showed red, blue, and green colorations, respectively. Apoptosis of MDAMB-231 cells was detected using the calcein-acetoxymethyl (AM)/propidium iodide (PI) apoptosis detection method. Briefly, cells were seeded in confocal dishes at the density of $1 \times 10^{5}$ cells per dish. At $80 \%$ confluency, drug-free DMEM, HMPBs, DOX, HMPBs-PFH, HMPBs-DOX, and HMPBsDOX/PFH were added to the cells, with and without focused ultrasound (FUS) for $20 \mathrm{~s}$ at $1 \mathrm{~W}$. After $2 \mathrm{~h}$ of incubation, cells were harvested and washed three times with PBS and were then stained with calcein-AM/PI for $10 \mathrm{~min}$ and observed under CLSM.

To quantitatively determine the cell apoptosis degree, we verified the apoptosis of MDA-MB-231 cells by flow cytometry (BD Biosciences, San Jose, CA, USA). MDAMB-231 cells were divided into the following groups: control group, FUS alone, HMPBs-DOX/PFH alone, HMPBs + FUS, HMPBs-PFH + FUS, and HMPBs-DOX/PFH + FUS. Except for the cells in the FUS group, all of these cells were, respectively, incubated with $\mathrm{HMPBs}$, HMPBs-PFH, and HMPBs-DOX/PFH containing the serum-free medium. After incubating for $3 \mathrm{~h}$, the treated MDA-MB-231 cells in each well were rinsed three times with PBS. Finally, all these cells apart from the cells in HMPBs-DOX/PFH alone were exposed to FUS for $20 \mathrm{~s}$ at $1 \mathrm{~W}$. After treatment for $24 \mathrm{~h}$, the apoptosis of cells was measured using Annexin-V fluorescein isothiocyanate (FITC)/PI double staining and analyzed by flow cytometry.

\section{In vitro ultrasound and MRI}

Approximately $2 \%$ agarose gel with columnar wells as a model was used for ultrasound imaging of HMPBs-DOX/ $\mathrm{PFH}$ in vitro. HMPBs-DOX/PFH were added into the wells and exposed to low-intensity FUS (LM.SC051 ACA; Institute of Ultrasound Imaging of Chongqing Medical Sciences, Chongqing, People's Republic of China) at $5 \mathrm{~W}$ for $30 \mathrm{~s}$. Next, the samples were immediately scanned in B-mode (brightness modulation) and contrast-enhanced ultrasound (CEUS) by a clinical ultrasonic diagnostic instrument (5-12 MHz linear probe, MyLab 90; Esaote, Genoa, Italy). Samples of pure PFH, HMPBs, and saline under the same conditions were also exposed and scanned, and the ultrasound images before and after exposure were observed. Mean echo intensity (mean decibel) was measured using DFY software (Institute of Ultrasound Imaging of Chongqing Medical Sciences).
A clean Eppendorf tube ( $2 \mathrm{~mL}$ in volume) was filled with HMPBs-DOX/PFH at different concentrations $(0.2,0.4,0.6$, 0.8 , and $1.0 \mathrm{mg} / \mathrm{mL}$ ) and covered to exclude all air. All the samples were imaged by a 7.0 T Bruker Biospec small animal MRI system (Bruker Corporation, Billerica, MA, USA). T2-weighted images were acquired using the following parameters: fast field echo, repetition time $(\mathrm{TR})=3.0 \mathrm{~s}$, echo time $(\mathrm{TE})=45 \mathrm{~ms}$, flip $=90^{\circ}$, field of view $(\mathrm{FOV})=40 \times 40 \mathrm{~mm}$, slice thickness $=1.0 \mathrm{~mm}$. The T2-weighted time of relaxation within the region of interest (ROI) was further measured.

\section{Ex vivo and in vivo experiments}

\section{Ex vivo experiments by HIFU exposure}

Fresh bovine liver was sliced into $12 \times 8 \times 6 \mathrm{~cm}^{3}$ pieces and degassed by vacuum equipment for $30 \mathrm{~min}$. After the degassing process, the bovine liver slices were placed in a container and immersed in degassed water. Saline, HMPBs, HMPBs-DOX, and HMPBs-DOX/PFH suspensions in $1 \times$ PBS $(200 \mu \mathrm{L})$ were directly injected into the targeted areas, which were monitored in real time using the ultrasound imaging unit of the HIFU therapeutic system. Immediately after the injection, HIFU was directed at the injection site with different HIFU parameters (120 W for $5 \mathrm{~s} ; 150 \mathrm{~W}$ for $5 \mathrm{~s} ; 180 \mathrm{~W}$ for $5 \mathrm{~s}$ ). Before and after HIFU exposure, the echo intensity of B-mode ultrasound images within the ROIs was accurately measured using the Gray Val 1.0 software affiliated with the HIFU system. After HIFU exposure, the length, width, and depth of necrotic tissues in the degassed bovine liver were measured to calculate their volumes in the bovine liver using the following formula: $\mathrm{V}=\pi / 6 \times \mathrm{L} \times \mathrm{W} \times \mathrm{D}$ (L, length; W, width; $\mathrm{D}$, depth).

\section{Implantation of VX2 liver tumors}

New Zealand white rabbits weighing 2.0-2.5 kg and between 2 and 3 months old were bred and housed between $19^{\circ} \mathrm{C}$ and $22^{\circ} \mathrm{C}$ and humidity conditions according to the institute's environmental guidelines. Food and water were provided ad libitum. All in vivo experiments were subjected to approval by the Institutional Animal Care and Use Committee of Chongqing Medical University. The experimental operations were carried out according to the protocol approved by the Institutional Animal Care and Use Committee of Chongqing Medical University.

Before establishing the animal model, the abdomens of rabbits were depilated with $8 \% \mathrm{Na}_{2} \mathrm{~S}$ solution. The tumor tissue was excised from a tumor-bearing rabbit, washed with saline, subdivided into small tissue pieces of about $1 \mathrm{~mm}^{3}$, and then soaked in $20 \mathrm{~mL}$ of Hanks' balanced salt solution for further use. Rabbits were anesthetized by intravenous 
injection of $3 \%$ pentobarbital solution $(1 \mathrm{~mL} / \mathrm{kg})$, fixed in the prone position, and routinely disinfected. After the middle lobe of liver below the xiphoid was revealed, a tissue piece was implanted into the liver using ophthalmological forceps. Before abdominal wall saturation, bleeding was stopped with gauze oppression. To prevent infection, 400,000 $\mathrm{U}$ of penicillin was intramuscularly injected for 3 days.

\section{In vivo ultrasound and MRI}

Then, 21 days after VX2 tumor inoculation, rabbits with detectable liver cancer were anesthetized and used for ultrasound and T2-weighted MRI experiments. The rabbits were injected with saline, HMPBs, HMPBs-DOX, or HMPBs-DOX/PFH $(10 \mathrm{mg} / \mathrm{mL})$ through intratumor injection $(300 \mu \mathrm{L})$. Ultrasound imaging with B-mode and CEUS was performed using a linear probe before injection, after injection, and after HIFU exposure. Likewise, MRI was performed before and after injection using a Philips Achieva 3.0 T MRI scanner (Philips Healthcare, Andover, MA, USA). The imaging parameters for T2-weighted imaging gradient echo were set at $\mathrm{TR}=2,500 \mathrm{~ms}, \mathrm{TE}=80 \mathrm{~ms}$, and flip angle $=45^{\circ}$. Images were obtained with a matrix size of $256 \times 256$, section thickness of $2 \mathrm{~mm}$, and FOV of $160 \times 160 \mathrm{~mm}$. Saline was used as the control under the same MRI parameters.

\section{In vivo HIFU ablation therapy}

New Zealand white rabbits with VX2 liver cancer were anesthetized, injected with saline, HMPBs, DOX, HMPBs-DOX, or HMPBs-DOX/PFH (10 mg/mL, $300 \mu \mathrm{L})$ intratumorally, and placed on the treatment couch in a prone position. The depilated area of the abdomen was completely immersed in the degassed water. The tumor was orientated and monitored by integrated diagnostic ultrasound imaging before HIFU exposure. The rabbits $(n=4)$ received an intratumor injection and were exposed to HIFU irradiation at $120 \mathrm{~W}$ for $5 \mathrm{~s}$. After HIFU exposure, the tumor volumes and tumor vascularity signal of all rabbits on days 1 and 7 were recorded using diagnostic ultrasound imaging. Similarly, saline was employed for the control group under the same HIFU therapeutic protocol. The animals were then euthanized, and tumor tissues were harvested from five groups of rabbits for staining with proliferating cell nuclear antigen (PCNA) for histopathological analysis by optical microscope. A homogeneous brown color of the nucleus indicates PCNA-positive cells. The positive cell count was semi-quantitatively observed by counting the number of cells stained positively in five random areas (400× magnification) in each slide. The proliferative index of PCNA = the number of PCNA-positive cells/total number of cells observed.

\section{Results and discussion Characterization of HMPBs-DOX/PFH}

The huge cavities, mesoporous structure, and cubic morphology of HMPBs can be directly observed in the TEM image (Figure 1A). Owing to the hollow mesoporous structure and large surface area, HMPBs are potentially ideal carriers to encapsulate anticancer drugs and/or other molecules. Moreover, the corresponding EDS mapping exhibited much weaker carbon, iron, and potassium element signal intensities $^{33}$ compared with the composition of the shell, which further indicated that HMPBs have been successfully synthesized by PB and formed the hollow mesoporous structure (Figure 1B). The well-dispersed HMPBs possessed an average dynamic diameter of $276.2 \mathrm{~nm}$, zeta potential of -12.3 $\mathrm{mV}$, and a particle dispersion index of 0.189 (Figure 1C and D), indicating that PVP and $\mathrm{HCl}$ can be used as a good protectant and etchant, respectively. The average diameter of the HMPBs-DOX/PFH at $37^{\circ} \mathrm{C}$ had no obvious change during $24 \mathrm{~h}$, which illustrated that the hybrid mesoporous nanoparticles had good stability (Figure S1). The phase transformation phenomenon of HMPBs-DOX/PFH was directly observed by optical microscopy at increasing temperatures (Figure 1E). Abundant small bubbles were released from HMPBs-DOX/PFH, while there were few gas bubbles in the HMPBs-DOX group, which provided strong evidence that PFH had been successfully encapsulated into the HMPBsDOX (Figure S2). The swelling and merging of a cluster of adjacent small microbubbles generated larger ones, which could explain the effects on ultrasound imaging and HIFU ablation in terms of the cavitation effect of stable bubbles released from the CAs and enhancement agents. These in vitro results demonstrate the potential of HMPBs-DOX/ PFH to substantially enhance ultrasound imaging.

\section{Doubly responsive triggered drug-release profile}

The loading efficiency of DOX was $87.5 \%$, and the loading capacity was $59.32 \%(w / w)$ in the HMPBs-DOX/PFH, as measured by HPLC. Such a significantly higher loading efficiency was mainly attributed to the special characteristics of the hollow mesoporous structure with its large cavity and numerous mesopores on the outer shell, and the presence of electrostatic interactions between HMPBs and DOX, as well as the strong coordinative bonding between inherent Fe(III) in the structure of HMPBs and chemical groups (eg, amino and carbonyl) of DOX at $\mathrm{pH}$ 7.4. The release behaviors of DOX from HMPBs-DOX/PFH in four groups (HIFU + pH 7.4 group, HIFU + pH 5.0 group, $\mathrm{pH} 7.4$ group, $\mathrm{pH} 5.0$ 
A

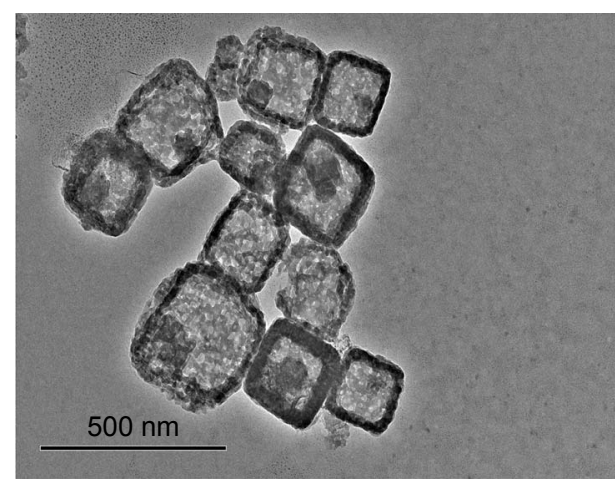

C

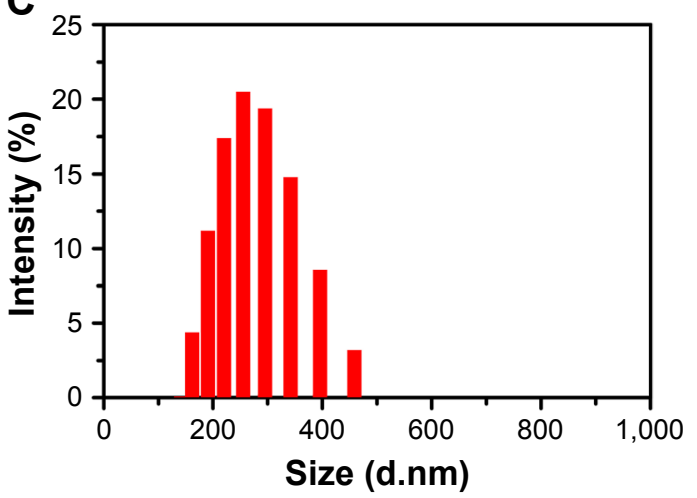

B

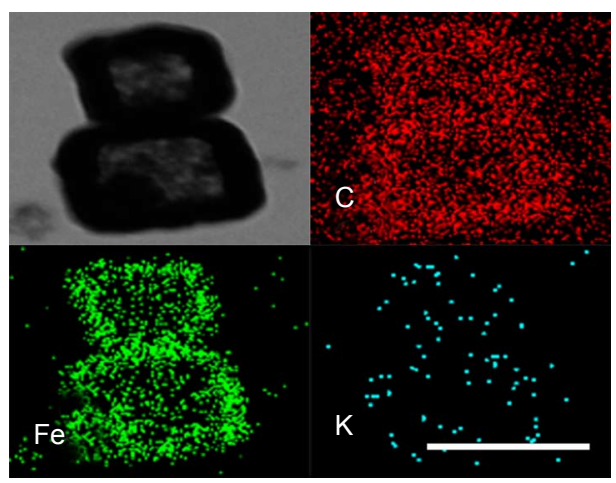

D

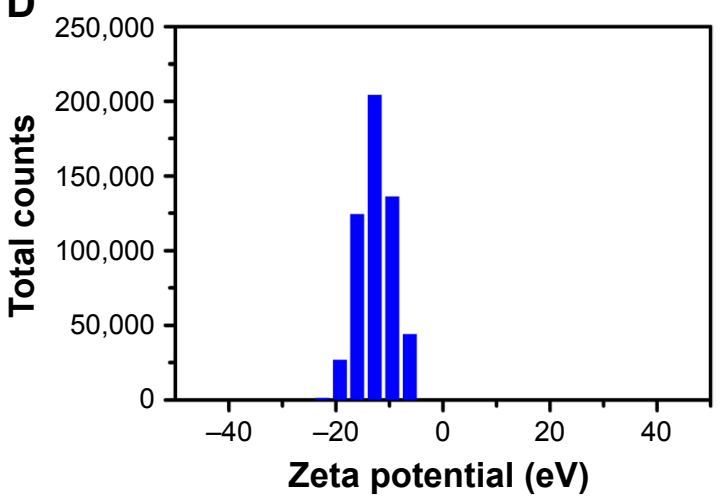

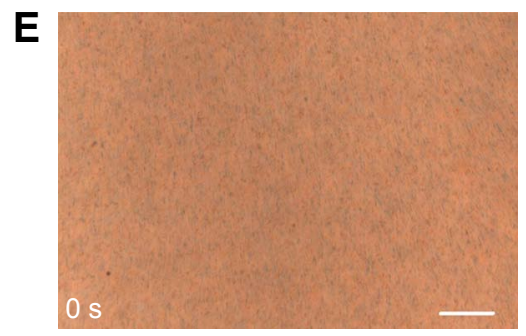
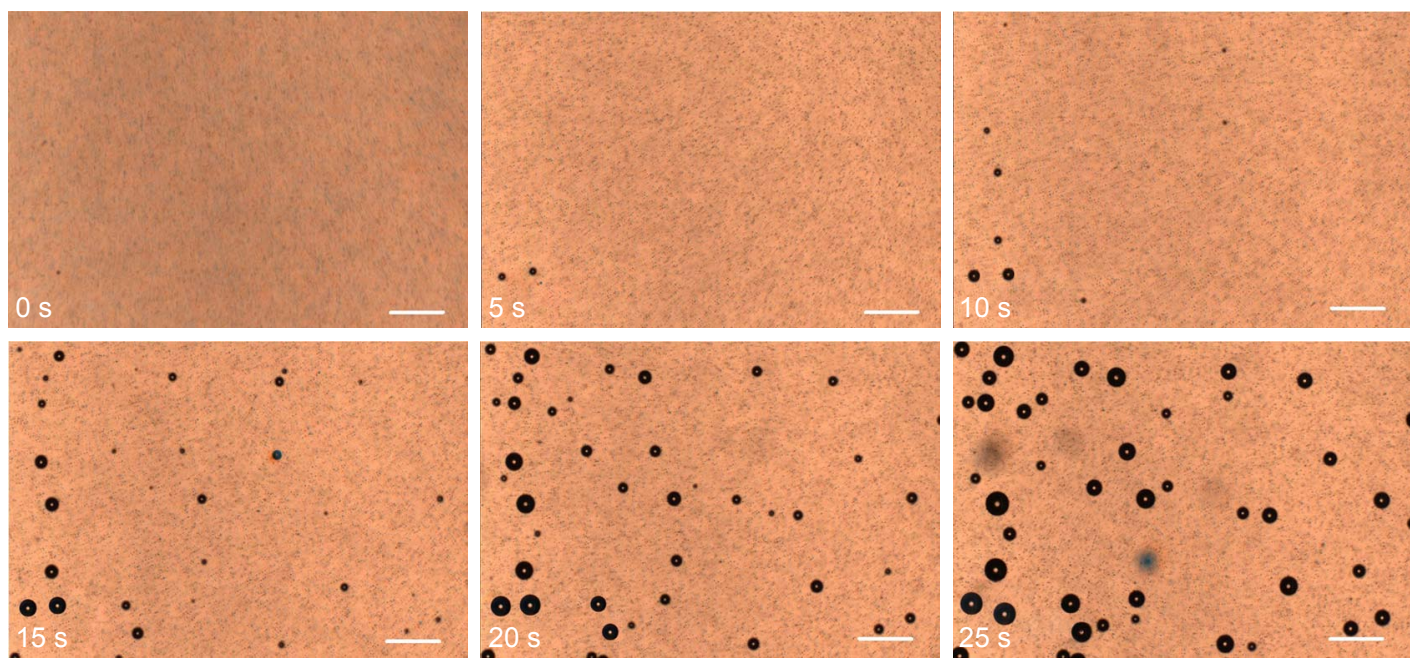

Figure I Morphology, structure, and characterizations of the HMPB nanoplatforms.

Notes: (A) A TEM image of HMPBs. (B) The magnification TEM images of HMPBs (upper left) and corresponding element mapping of carbon (C, upper right), iron ( $\mathrm{Fe}$, lower left), and potassium (K, lower right). The scale bar in (B) indicates $200 \mathrm{~nm}$. Magnification $\times 50,000$. (C, D) The size distribution and zeta potential in double-distilled water by DLS measurement. (E) Optical microscopic images of HMPBs-DOX/PFH nanoplatforms after heating at $65^{\circ} \mathrm{C}$. The scale bars in (E) indicate $50 \mu \mathrm{m}$. Magnification $\times 400$.

Abbreviations: DLS, dynamic light scattering; DOX, doxorubicin; HMPBs, hollow mesoporous Prussian blue nanoparticles; PFH, perfluorohexane; TEM, transmission electron microscope; HMPBs-DOX/PFH, DOX/PFH-encapsulated HMPBs.

group) were measured by HPLC to evaluate the effects of HIFU and $\mathrm{pH}$ values on DOX release (Figure 2A). The DOX release percentage increased from $12.07 \%$ to $48.22 \%$ at pH 5.0 in $48 \mathrm{~h}$, while it increased from $10.55 \%$ to $15.62 \%$ at $\mathrm{pH} 7.4$; this can be ascribed to linkages/bonds between DOX and the effective combination sites of HMPBs, which will be weakened at reduced pH values (Figure 2B).
Therefore, with ultrahigh relaxivity sensitive to $\mathrm{pH}$, this nanoplatform can intelligently release an anticancer drug at the tumor site to enhance synergistic chemotherapy, which is of great significance for amplifying therapeutic efficacy, decreasing systemic side effects, and reducing the possibility of tumor recurrence. In addition, slow release was found as expected for the reference samples of HMPBs-DOX/PFH 
A

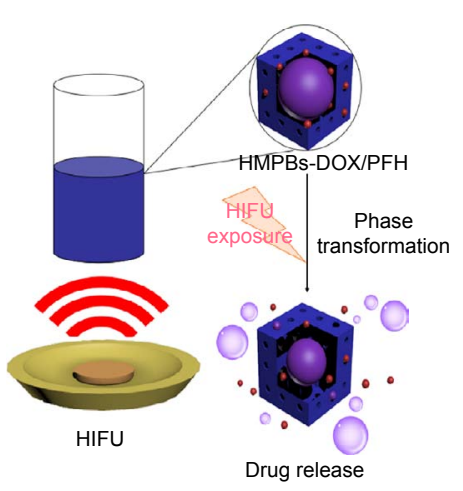

B

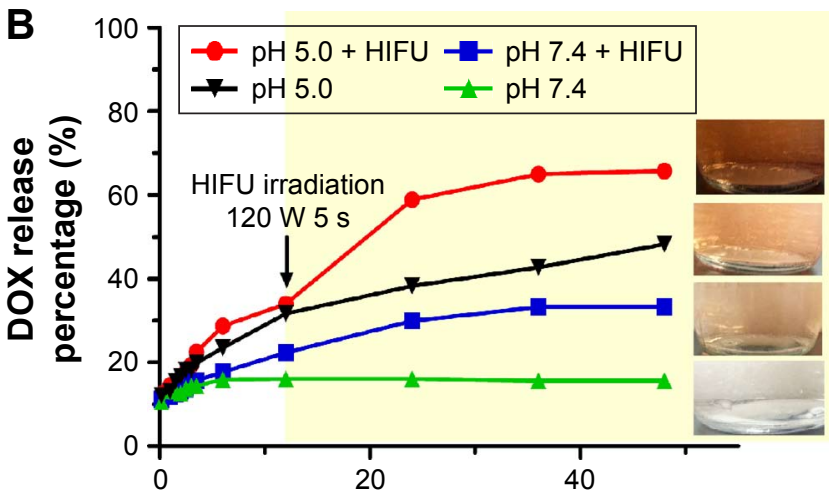

Time (h)

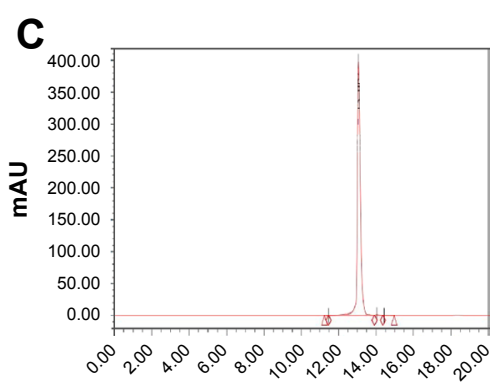

Control

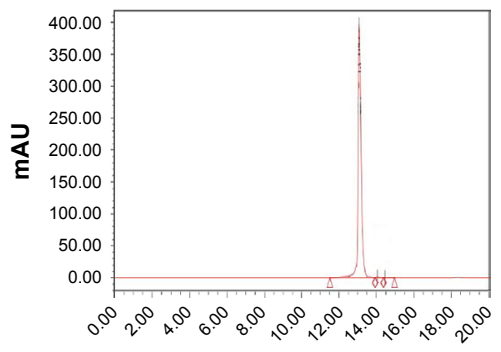

$80^{\circ} \mathrm{C}, 15 \mathrm{~min}$

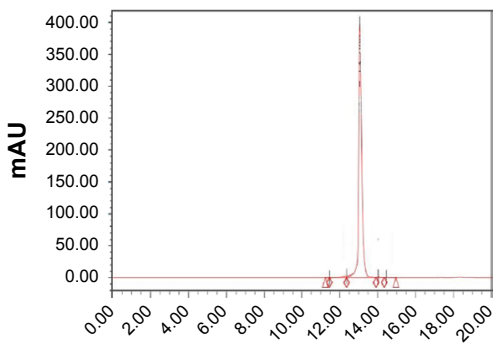

$80^{\circ} \mathrm{C}, 30 \mathrm{~min}$

Figure 2 In vitro release of DOX from HMPBs-DOX/PFH by double stimuli.

Notes: (A) A schematic of HIFU-triggered DOX release from HMPB-DOX/PFH for chemotherapy. (B) The drug release percentage curve of HMPBs-DOX/PFH at pH 5.0 and 7.4 with and without HIFU irradiation. Insets: digital photos of drug concentration with different treatments at $48 \mathrm{~h}$ time point. (C) HPLC images of DOX solution at different temperatures (room temperature, $80^{\circ} \mathrm{C}$ for $15 \mathrm{~min}$, and $80^{\circ} \mathrm{C}$ for $30 \mathrm{~min}$, respectively).

Abbreviations: DOX, doxorubicin; HIFU, high-intensity focused ultrasound; HMPBs, hollow mesoporous Prussian blue nanoparticles; HPLC, high-performance liquid chromatography; PFH, perfluorohexane; HMPBs-DOX/PFH, DOX/PFH-encapsulated HMPBs.

(with $33.95 \%$ and $22.31 \%$ drug release from carriers at $\mathrm{pH} 5.0$ and 7.4, respectively, after $12 \mathrm{~h}$ ) before HIFU irradiation. Interestingly, the cumulative release amounts were $65.76 \%$ at $\mathrm{pH} 5.0$ and $33.20 \%$ at $\mathrm{pH} 7.436 \mathrm{~h}$ post-irradiation $(120 \mathrm{~W}$, $5 \mathrm{~s})$. The irradiation of HIFU can accelerate the release of DOX from HMPBs-DOX/PFH, which was significantly different compared with the DOX release at different $\mathrm{pH}$ values in the control group. It just took $24 \mathrm{~h}$ for $50 \%$ of DOX to be released from the nanoplatforms after irradiation in the HIFU group, whereas $\sim 65.76 \%$ of DOX was released after $48 \mathrm{~h}$ post-irradiation. However, only about $33.20 \%$ of the encapsulated DOX was released in the control group at the time point of $48 \mathrm{~h}$. This dramatic and rapid increase in the amount of drug release is attributed to the evaporated $\mathrm{PFH}$ gas under thermal effects, as well as the cavitation effects. We also verified the difference of drug release efficiency between HMPBs-DOX/PFH and HMPBs-DOX under the same HIFU irradiation and found that PFH-loaded nanoplatform could release more drugs than HMPBs-DOX. The reason for this phenomenon was that liquid PFH would be converted into a large population of small bubbles and released in a sustained manner via the mesopore channels of HMPBs after exposure to HIFU, which resulted in greatly improved drug release.
Moreover, the destruction of generated bubbles may cause high-energy microstreams or microjets that could facilitate the dispersion of drug molecules in situ. This result illustrated that PFH played an important role in the drug release (Figure S3). According to the in vitro results, HMPBs-DOX/ PFH can be developed as a good drug delivery system, the features of which include responsive drug release based on $\mathrm{pH}$ and HIFU irradiation, to improve the drug bioavailability, minimize the side effect of chemotherapeutics to normal tissues, and enhance the antitumor efficiency. In addition, the thermostability of DOX at high temperatures was validated by HPLC. As shown in Figure 2C, HPLC traces of DOX solution after incubation at $80^{\circ} \mathrm{C}$ for $15 \mathrm{~min}$ or $30 \mathrm{~min}$ were the same as at room temperature, indicating that the drug properties of DOX are preserved after HIFU irradiation.

\section{In vitro cytotoxicity and antitumor efficacy of HMPBs-DOX/PFH}

The cytotoxicity of HMPBs was observed for MDA-MB-231 breast carcinoma cells at different HMPB concentrations. No significant cytotoxicity of HMPBs was observed for MDA-MB-231 breast cancer cells, even at a high concentration of $400 \mu \mathrm{g} / \mathrm{mL}$ (Figure 3A), indicating that HMPBs are 
biologically safe as nanoplatforms. We further determined the therapeutic efficacy of the acoustic effect and chemotherapy for killing the human breast carcinoma cell line MDA-MB-231. Relative cell viabilities were investigated by the apoptosis detection with calcein-AM/PI using CLSM. We first confirmed that the nanoplatforms were internalized within the cell and not on the cell membrane. As shown in Figure $3 \mathrm{~B}$, it could be directly observed that large amounts of HMPBs-DOX/PFH (DOX showed red fluorescent dots) were present in the cytoplasm of the MDA-MB-231 cells, which had been incubated with $100 \mu \mathrm{g} / \mathrm{mL}$ of HMPBs-DOX/ PFH for $2 \mathrm{~h}$. The cells were stained with DAPI to mark the nucleus (blue fluorescence) and DiO to mark the cell membrane (green fluorescence). The merged imaging gives direct evidence of the phagocytosis efficiency of HMPBs-DOX/ PFH. CLSM images of the MDA-MB-231 cells were also taken to visually differentiate the live cells (green) from the dead cells (red) in different treatment groups (Figure 3C). After FUS irradiation for a limited time, the cell viability after various treatments was lower than the cell viability without FUS irradiation. PFH-encapsulated nanoplatforms resulted in significantly high rates of apoptosis as compared with other groups after FUS irradiation. Particularly, the FUSmediated HMPBs-DOX/PFH group exhibited a higher rate of cell apoptosis, even higher than the FUS-mediated DOX cancer drug group. The cytotoxic effect of HMPBs-DOX/ PFH on MDA-MB-231 cells was further quantified by flow cytometry. The apoptosis efficiency for the FUS-mediated HMPBs-PFH and HMPBs-DOX/PFH groups experienced the conspicuous cell apoptosis, while the HMPBs-DOX/ $\mathrm{PFH}$ alone group's apoptosis rate was lower than the HMPBs-PFH + FUS and HMPBs-DOX/PFH + FUS groups.
A

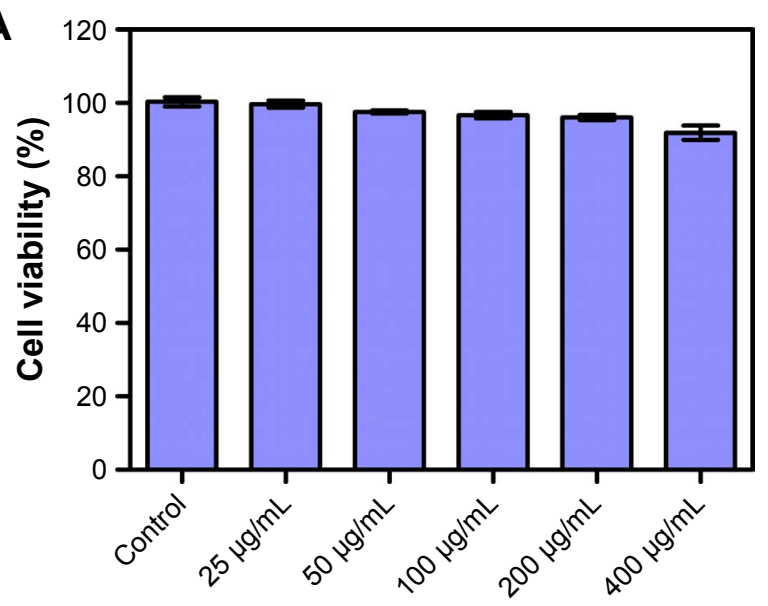

B
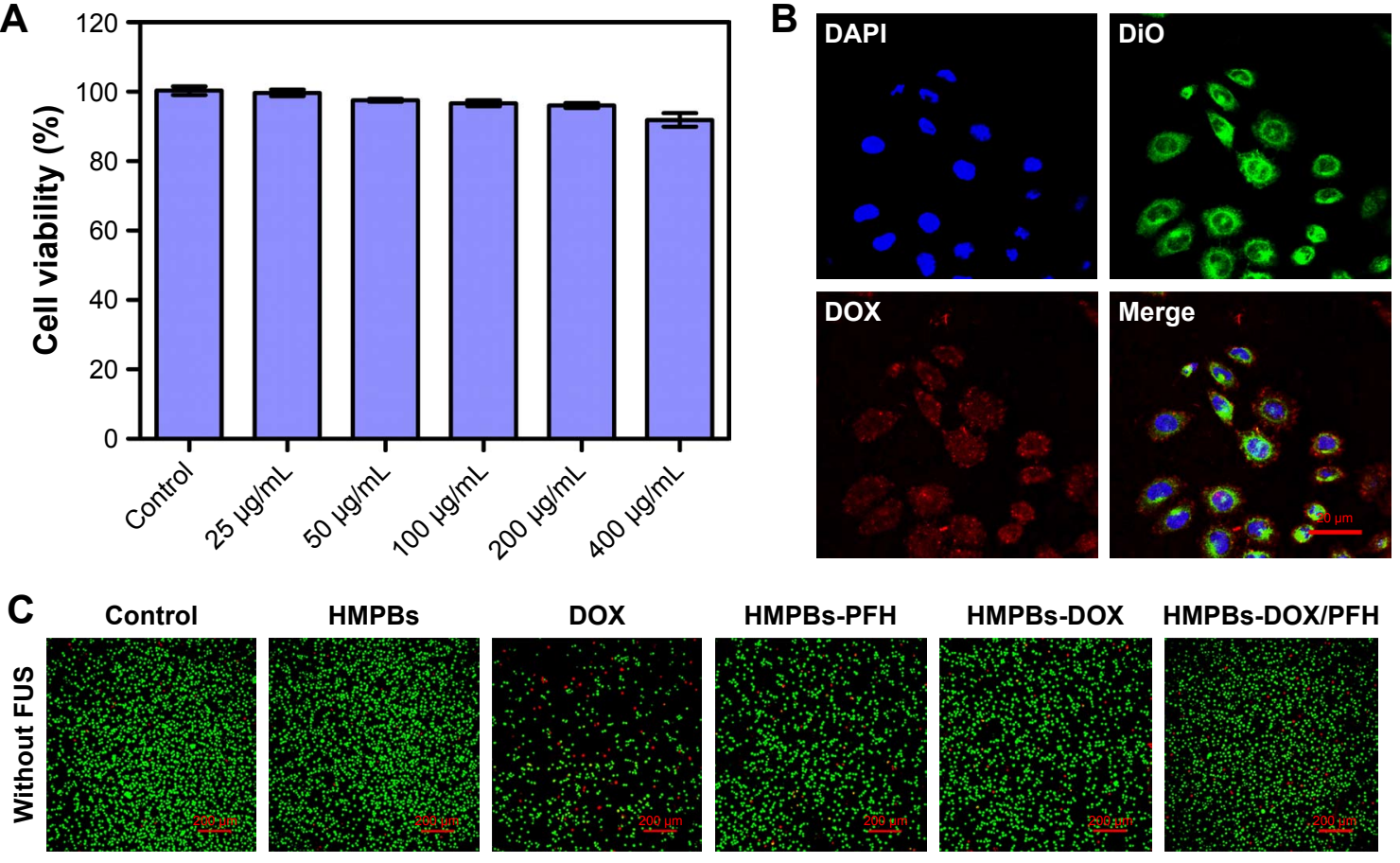

HMPBs-DOX/PFH
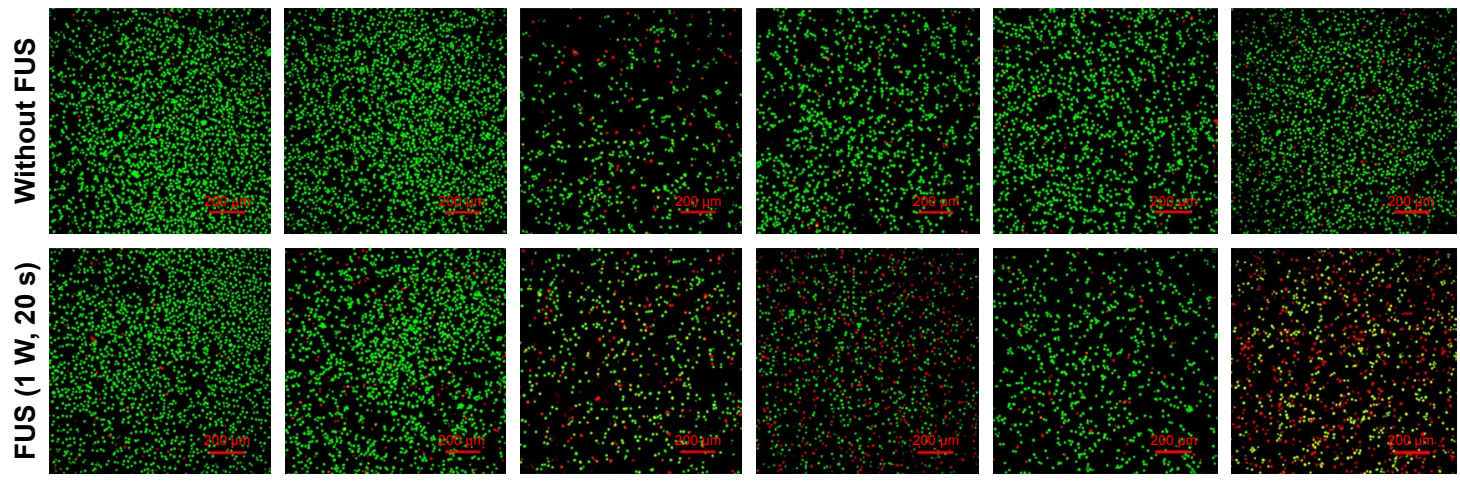

Figure 3 In vitro cytotoxicity and antitumor efficacy of HMPBs-DOX/PFH.

Notes: (A) Cell viabilities of HMPBs solutions at various concentrations (0, 25, 50, 100, 200, and $400 \mu g / \mathrm{mL})$. (B) CLSM image of MDA-MB-23I cells for cytophagy experiment with HMPBs-DOX/PFH at $100 \mu \mathrm{g} / \mathrm{mL}$. DAPI-labeled cell nuclei (blue fluorescence, upper left), DiO-labeled cell membrane (green fluorescence, upper right), DOX-loaded HMPBs-DOX/PFH (red fluorescence, lower left), and merge imaging (lower right). The scale bars in (B) indicate $20 \mu \mathrm{m}$. Magnification $\times 800$. (C) CLSM images of cytotoxicity test for MDA-MB-23I cells with different treatments via PI and AM-calcein double labeling, wherein apoptotic cells (red color) were stained by PI, and viable cells (green color) were stained by AM-calcein. The scale bars in (C) indicate $200 \mu \mathrm{m}$. Magnification $\times 100$.

Abbreviations: AM, acetoxymethyl; CLSM, confocal laser scanning microscopy; DAPI, 2-(4-amidinophenyl)-6-indolecarbamidine dihydrochloride; DiO, 3,3'-dioctadecyloxacarbocyanine perchlorate; DOX, doxorubicin; FUS, focused ultrasound; HMPBs, hollow mesoporous Prussian blue nanoparticles; PFH, perfluorohexane; PI, propidium iodide; HMPBs-DOX/PFH, DOX/PFH-encapsulated HMPBs. 
Furthermore, FUS alone, HMPBs + FUS group barely showed damage to the cells (Figure S4). These results demonstrate that this nanoplatform can be vaporized to generate bubbles by the acoustic effect of FUS. The subsequent post-generated bubbles can substantially change the acoustic microenvironment and generate a series of cavitation-related bioeffects, such as direct mechanical oscillation, cell membrane opening, and free radical release, which damage the cells and enhance the uptake and release of the anticancer drugs in the tumor site during the limited irradiation time. Our in vitro study confirmed the synergistic effect between chemotherapy and FUS therapy, which is highly beneficial for improving the therapeutic efficacy of further cancer treatment. It is a reasonable conclusion that HMPBs-DOX/ PFH can optimize the therapeutic effects.

\section{In vitro ultrasound and $M R I$ of HMPBs-DOX/PFH}

The outstanding in vitro therapeutic efficacy of HMPBs-DOX/ $\mathrm{PFH}$ prompted us to investigate the imaging effect in more detail. Building on the results of the aforementioned in vitro phase transformation experiments, ultrasound imaging technology consisting of B-mode imaging and CEUS imaging modes was employed to monitor PFH bubble generation and behaviors. As shown in Figure 4A, as the negative control, the degassed saline group exhibited much lower echo intensity in both B-mode and CEUS, before and after HIFU irradiation. The control group of pure HMPBs was not able to produce microbubbles after HIFU irradiation. As a result, there was a lack of obvious changes in ultrasound signals between the degassed saline and the suspension of HMPBs before and after exposure to the same HIFU intensity. To convincingly evaluate whether pure PFH can generate microbubbles to enhance ultrasound imaging, we detected pure $\mathrm{PFH}$ in B-mode and CEUS before and after HIFU irradiation. Interestingly, there was no obvious contrast enhancement in both B-mode and CEUS, which further demonstrated that pure PFH could not generate microbubbles under HIFU irradiation. A significant contrast enhancement was observed in HMPBs-DOX/PFH suspension phase of the B-mode and the CEUS imaging, which can possibly be attributed to the cavitation effect induced by HIFU irradiation, causing phase change of PFH in the core. The quantitative echo intensities of the samples were acquired (Figure 4B and C). Compared with saline, pure PFH molecules, and pure HMPBs, there was a significant difference in the mean echo intensities of HMPBs-DOX/PFH $(p<0.05)$. The echo intensity of HMPBs$\mathrm{DOX} / \mathrm{PFH}$ was nearly sixfold greater than that of the other groups after irradiation. The signal intensity of CEUS in the HMPBs-DOX/PFH group was more than 10-fold that of other nanoplatform groups. These phenomena resulted from the swelling and merging of a cluster of neighboring small PFH microbubbles to generate larger ones, which could be directly observed by prolonging the irradiation duration. Microbubbles are known to alter surface tension, boost acoustic impedance, and enhance detectable scattering, which would result in intensified scattering signals and, consequently, enhanced ultrasound imaging. However, the encapsulated DOX in the core did not influence the ultrasound imaging capability of HMPBs-DOX/PFH. Therefore, the in vitro HIFU irradiation results demonstrate that the as-synthesized HMPBs-DOX/PFH nanoplatform can act as a phase change SA for enhancing HIFU cancer treatment efficiency and monitoring therapeutic effect.

MRI is an external diagnostic imaging technique which currently uses HIFU guidance to identify the lesion tissues. To evaluate the MRI guiding capabilities of HMPBs-DOX/ $\mathrm{PFH}$, transverse relaxivity was measured using a $7.0 \mathrm{~T}$ Bruker Biospec small animal MRI system. As revealed by the MRI phantom images (Figure 4D), HMPBs-DOX/PFH are negatively enhanced in T2-weighted MRI. The MRI transverse photon relaxation time decreased with increasing HMPB concentration, which enhanced the T2-weighted MRI images (Figure 4E). This could account for the effects of nanoplatforms containing the superparamagnetic iron ion, which also generate a negative contrast-enhanced effect for T2-weighted MRI. Overall, these results demonstrate the in vitro efficiency of the HMPBs-DOX/PFH as a dualmodality ultrasound/MRI CA for the guidance and monitoring of HIFU ablation therapy.

\section{In vivo ultrasound and $M R I$ of HMPBs-DOX/PFH}

To assess whether HMPBs-DOX/PFH could be developed for in vivo ultrasound imaging, further animal experiments were performed in VX2 tumor-bearing rabbits. A total of 20 tumor-bearing rabbits were divided into four groups of five and injected intratumorally with the same concentration of HMPBs-DOX/PFH, HMPBs-DOX, HMPBs, and saline, respectively. As shown in Figure 5A, red dotted lines indicated the shape of VX2 tumors, and the ROIs were specified as large as the injection positions in the VX2 tumors. There were no obvious changes in either B-mode or CEUS imaging after HIFU irradiation was observed in the saline, HMPBs, or HMPBs-DOX group. However, ultrasound imaging of the rabbit liver tumor injected with HMPBs-DOX/PFH 
A

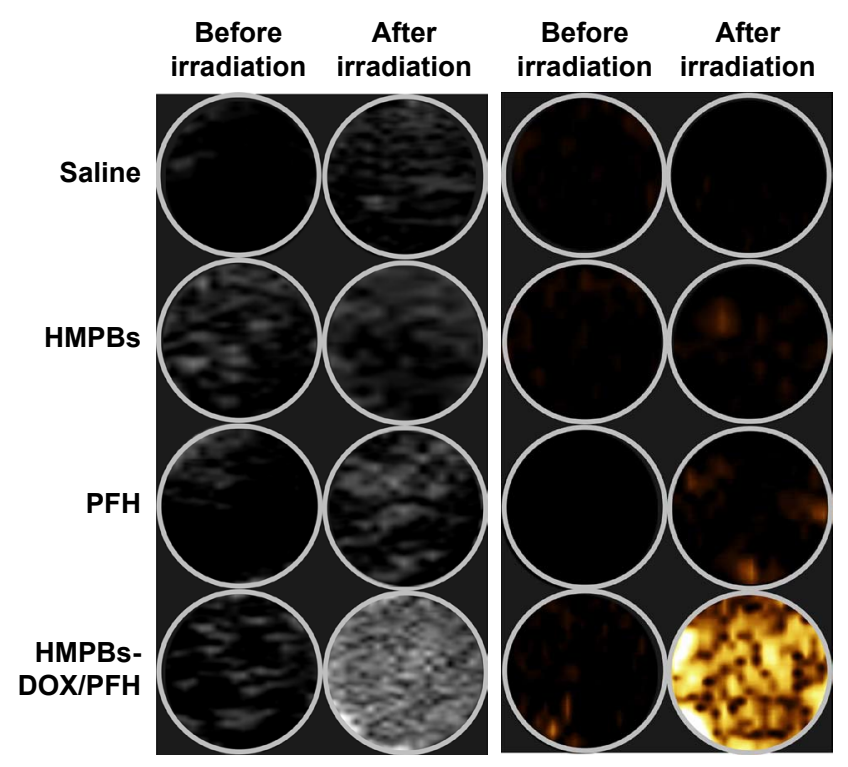

D

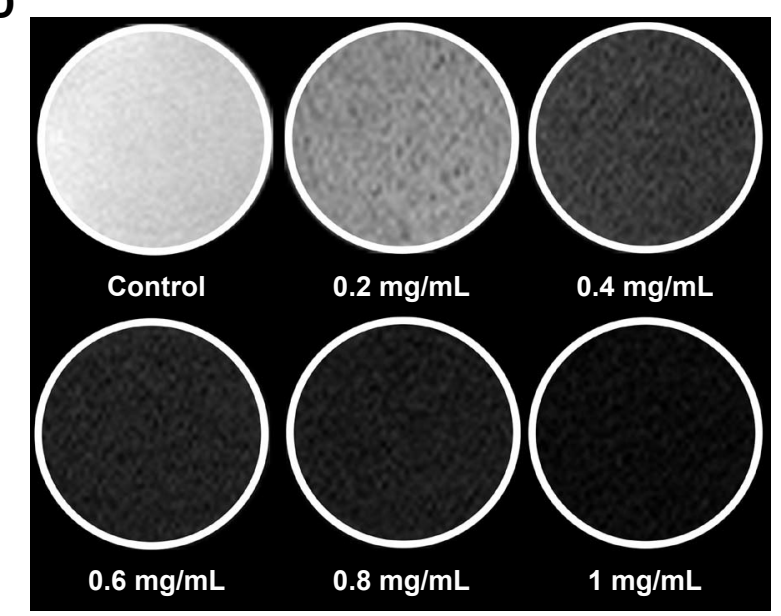

B

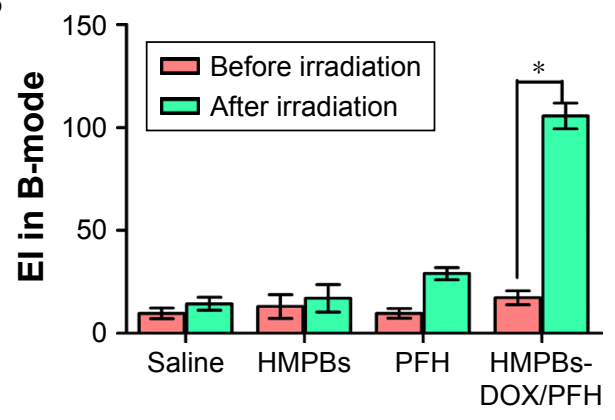

C

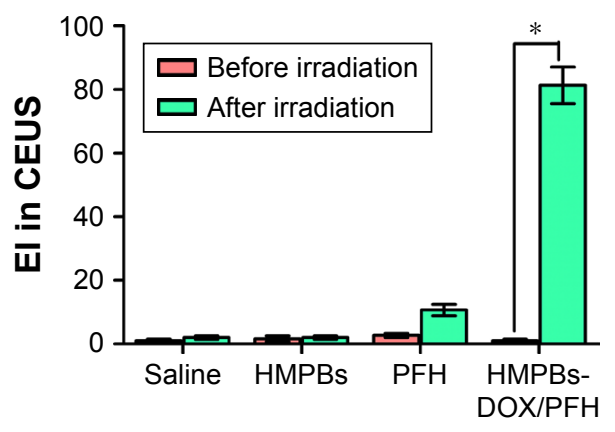

E

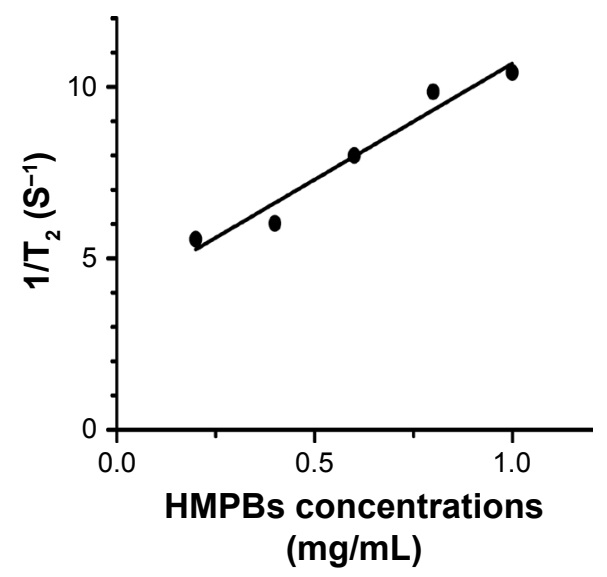

Figure 4 In vitro dual-modality US/MRI results.

Notes: (A) B-mode and CEUS images before and after irradiation in vitro (gel model) for saline, HMPBs, PFH, and the same concentration of HMPBs-DOX/PFH (top row, second row, third row, and last row, respectively). (B, C) Corresponding quantitative El of B-mode and CEUS imaging before and after irradiation. *Signifcant differences in $\mathrm{El}$ in B-mode and CEUS by comparing each group at a statistical $p$-value $(p<0.05)$. (D) T2-weighted images of HMPBs-DOX/PFH at different concentrations under a 7.0 T magnetic field. (E) The relativity between transverse relaxivities $\left(r_{2}\right)$ and various concentrations of ROls (white circle).

Abbreviations: B-mode, brightness modulation; CEUS, contrast-enhanced ultrasound; DOX, doxorubicin; El, echo intensity; HMPBs, hollow mesoporous Prussian blue nanoparticles; MRI, magnetic resonance imaging; PFH, perfluorohexane; ROls, regions of interest; US, ultrasound; HMPBs-DOX/PFH, DOX/PFH-encapsulated HMPBs.

before HIFU irradiation exhibited a low gray-scale level, while the ultrasonic signal of the tumor region increased significantly after HIFU irradiation. Correspondingly, the quantitative echo intensities of the injection positions were acquired (Figure 5B). Compared with the saline, HMPBs, and HMPBs-DOX groups, the mean echo intensities before and after irradiation were significantly different in the case of HMPBs-DOX/PFH $(p<0.05)$. This demonstrates that PFH has a temperature-responsive effect and generates a cluster of PFH microbubbles, which could enhance the capability of ultrasound imaging in B-mode and CEUS. These results demonstrated that HMPBs-DOX/PFH has great potential for imaging-guided HIFU treatment to monitor and assess the therapeutic efficacy, by providing more imaging information about the ablation lesion after HIFU treatment.

We further determined the imaging ability of HMPBsDOX/PFH as a CA for in vivo MRI. A remarkable negative contrast enhancement of T2-weighted MRI with HMPBsDOX/PFH can be observed since it could shorten the transverse relaxation, while the control group of saline did 

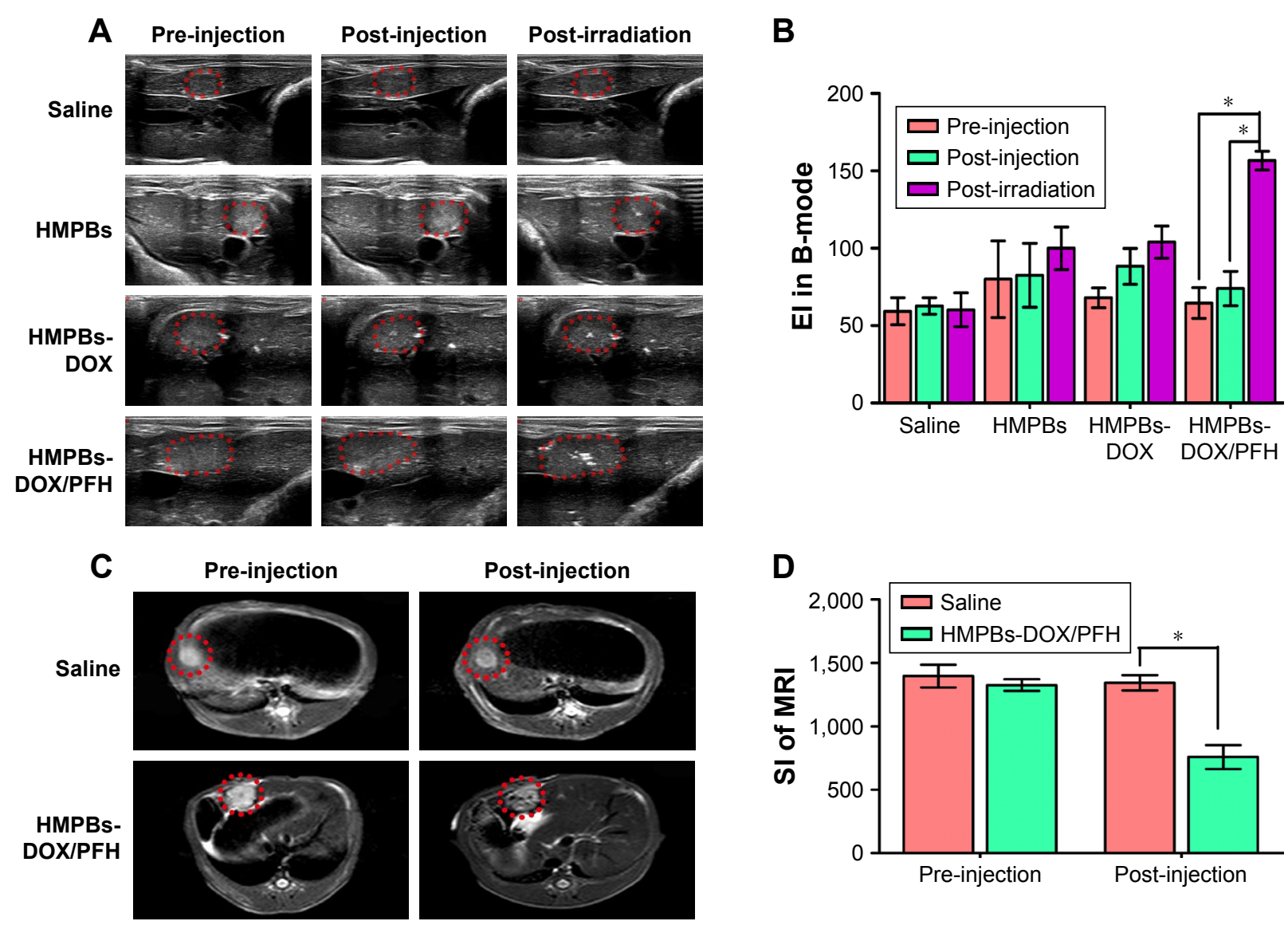

Figure 5 In vivo dual-modality US/MRI results.

Notes: (A) US imaging of rabbits bearing VX2 tumors before and after intratumoral injection of different solutions (saline, HMPBs, HMPBs-DOX, and HMPBs-DOX/PFH) and after HIFU irradiation. Red dotted lines indicate the shape of VX2 tumors. (B) Corresponding average gray values of injection positions in the VX2 tumors before injection, after injection, and after irradiation. *Significant differences in El in B-mode by comparing each group at a statistical $p$-value ( $p<0.05)$. (C) In vivo T2-weighted MRI and (D) their corresponding signal intensities for rabbits bearing VX2 tumors on the livers before and after intratumor HMPBs-DOX/PFH injection. HMPBs-DOX, DOXencapsulated HMPBs; HMPBs-DOX/PFH, DOX/PFH-encapsulated HMPBs. *Significant differences in SI of MRI by comparing each group at a statistical $p$-value ( $<<0.05)$.

Abbreviations: B-mode, brightness modulation; DOX, doxorubicin; El, echo intensity; HIFU, high-intensity focused ultrasound; HMPBs, hollow mesoporous Prussian blue nanoparticles; MRI, magnetic resonance imaging; PFH, perfluorohexane; SI, signal intensity; US, ultrasound.

not show any such effect (Figure 5C), further indicating the versatility and functionality of HMPBs-DOX/PFH. Specifically, the T2-weighted MRI signal intensity of the tumor with injection of HMPB-DOX/PFH decreased from $1,343.79 \pm 60.23$ to $758.35 \pm 94.53$, which is much lower than that observed for saline (Figure 5D), confirming that HMPBDOX/PFH is an excellent T2-weighted MRI CA in vivo. This ultrasound/MRI dual-modality biological imaging capability of HMPBs-DOX/PFH will be very beneficial for imaging-guided HIFU cancer surgery.

\section{Ex vivo HIFU synergistic effect assessment}

HMPBs-DOX/PFH as the SA for HIFU ablation was first evaluated in an ex vivo experiment, using degassed bovine liver as the model tissue. As shown in Figure 6A, the coagulative volume increased with increasing HIFU acoustic power $(120 \mathrm{~W}, 150 \mathrm{~W}$, and $180 \mathrm{~W})$. Importantly, HMPBs-DOX/ PFH with phase-changing capability could cause substantially larger tissue ablation volume compared with HMPBs-DOX, HMPBs, and saline as the control $(p<0.05$, Figure 6B). Compared with the saline samples $\left(4.96 \pm 1.76 \mathrm{~mm}^{3}\right.$ at $120 \mathrm{~W}$; $19.63 \pm 1.98 \mathrm{~mm}^{3}$ at $150 \mathrm{~W} ; 33.45 \pm 2.81 \mathrm{~mm}^{3}$ at $180 \mathrm{~W}$ ), HMPB samples $\left(17.04 \pm 2.39 \mathrm{~mm}^{3}\right.$ at $120 \mathrm{~W} ; 25.31 \pm 4.85 \mathrm{~mm}^{3}$ at $150 \mathrm{~W} ; 53.37 \pm 5.40 \mathrm{~mm}^{3}$ at $180 \mathrm{~W}$ ), and HMPBs-DOX samples $\left(14.72 \pm 3.72 \mathrm{~mm}^{3}\right.$ at $120 \mathrm{~W} ; 25.64 \pm 7.04 \mathrm{~mm}^{3}$ at $150 \mathrm{~W} ; 71.61 \pm 8.61 \mathrm{~mm}^{3}$ at $180 \mathrm{~W}$ ), a significant necrotic volume enlargement was found for the HMPBs-DOX/PFH samples $\left(38.78 \pm 3.28 \mathrm{~mm}^{3}\right.$ at $120 \mathrm{~W} ; 120.81 \pm 12.76 \mathrm{~mm}^{3}$ at $150 \mathrm{~W} ; 215.59 \pm 18.85 \mathrm{~mm}^{3}$ at $\left.180 \mathrm{~W}\right)$. These results demonstrated that the intervention of phase-changing nanoplatforms could generate bubbles and change tissue acoustic impedance, and thus increase the HIFU energy deposition and improve the efficiency of HIFU surgery. Furthermore, the corresponding B-mode ultrasound images at the focus before and after HIFU irradiation were evaluated quantitatively. As shown in Figure 6C, there was no apparent difference 
A
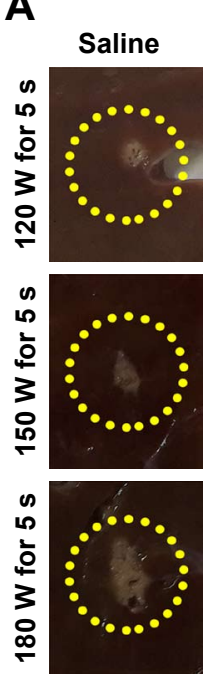

C

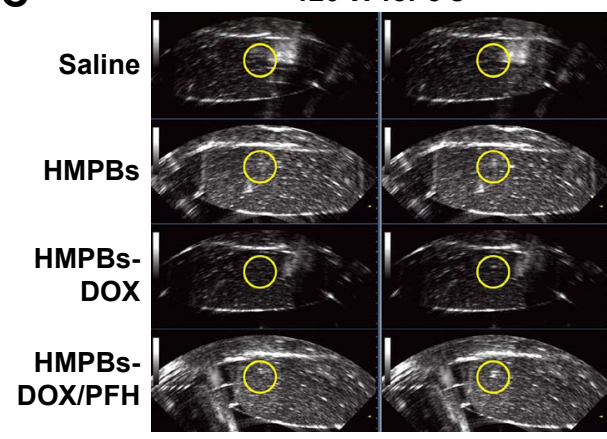

HMPBs- HMPBsDOX/PFH
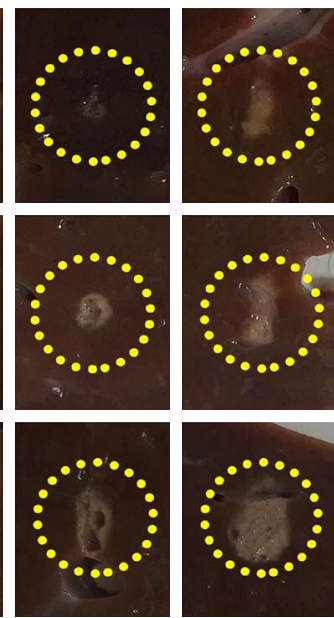

B

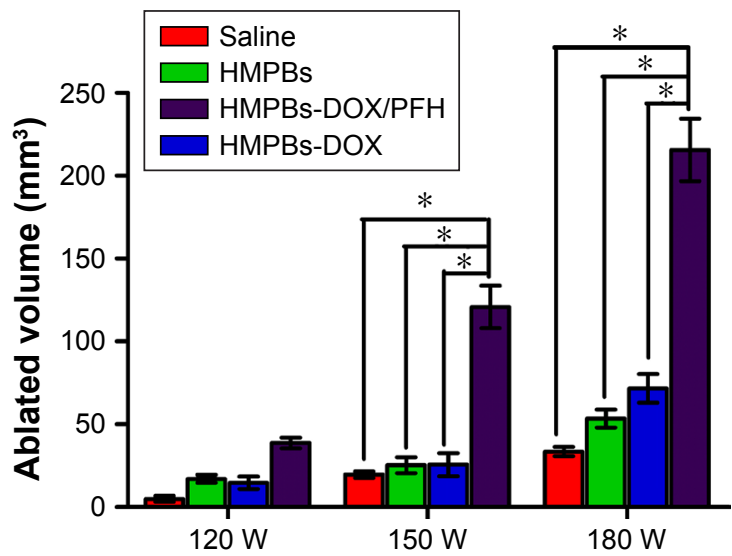

Figure 6 Ex vivo evaluation of the effectiveness of HMPBs-DOX/PFH as the HIFU therapeutic agent.

Notes: (A) Photograph of degsassed bovine liver exposed to HIFU ablation after the intratissue injection of various agents: saline, pure HMPBs, HMPBs-DOX, and HMPBsDOX/PFH. The HIFU irradiation parameters were $120 \mathrm{~W}$ for $5 \mathrm{~s}, 150 \mathrm{~W}$ for $5 \mathrm{~s}$, and $180 \mathrm{~W}$ for $5 \mathrm{~s}$. (B) The coagulation volume of the bovine liver after the injection of different samples and exposure to HIFU ablation. *Significant differences in coagulation volume by comparing each group at a statistical $p$-value $(p<0.05)$. (C) The B-mode ultrasound images at the focus in the degassed bovine liver injected with saline, pure HMPBs, HMPBs-DOX, and HMPBs-DOX/PFH before and after HIFU irradiation at I20, I50, and $180 \mathrm{~W}$ for $5 \mathrm{~s}$. Yellow cycles indicate the focus area of HIFU irradiation.

Abbreviations: B-mode, brightness modulation; DOX, doxorubicin; HIFU, high-intensity focused ultrasound; HMPBs, hollow mesoporous Prussian blue nanoparticles; PFH, perfluorohexane; HMPBs-DOX, DOX-encapsulated HMPBs; HMPBs-DOX/PFH, DOX/PFH-encapsulated HMPBs.

in the gray-scale value for the liver samples injected with HMPBs-DOX/PFH, HMPBs-DOX, HMPBs, and saline before HIFU irradiation. Interestingly, a remarkable contrast enhancement was observed in those samples injected with HMPBs-DOX/PFH after HIFU irradiation, while other groups exhibited limited differences after irradiation. These ex vivo findings indicated that the HMPBs-DOX/PFH have the potential to serve as HIFU SAs.

\section{HMPBs-DOX/PFH for synergistic HIFU liver cancer surgery}

The HIFU synergistic enhancement and associated synergistic mechanism were systematically investigated as anticancer therapy agents in vivo on rabbit liver tumor. The tumor was treated at HIFU power of $120 \mathrm{~W}$ and exposure duration of $5 \mathrm{~s}$. The development of the tumor and the HIFU ablation effect were observed by real-time ultrasound imaging with a clinical instrument. As shown in Figure 7A, the liver cancer treated with the HMPBs-DOX/PFH showed a significant decrease in growth volume compared with the control saline, HMPBs, DOX, and HMPBs-DOX. In the HMPBs-DOX/PFH group, significant differences were observed in the ultrasound images, which were attributed to tissue damage and necrosis. Blood flow was also reduced within the cancer tissue at day 7. The groups treated with saline, HMPBs, DOX, and HMPBs-DOX showed no significant changes throughout the treatment. It is well known that HIFU surgery can generate thermal and cavitation effects, acoustic streaming, and shear stresses, which induce coagulation necrosis. HMPBs-DOX/ PFH nanocapsules can generate microbubbles upon HIFU irradiation under a high-energy FUS beam. This procedure could be used to change the acoustic environment of tumor tissues, enhance the acoustic energy deposition, and decrease energy consumption to improve the HIFU therapeutic 

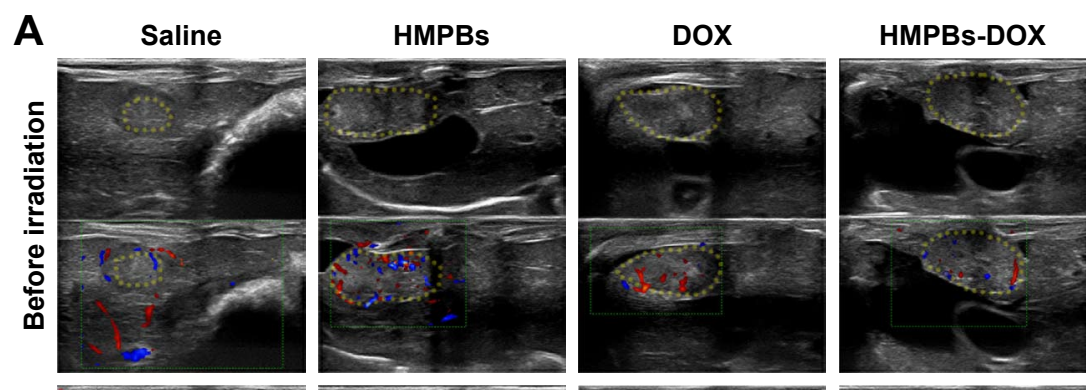

HMPBs-DOX/PFH
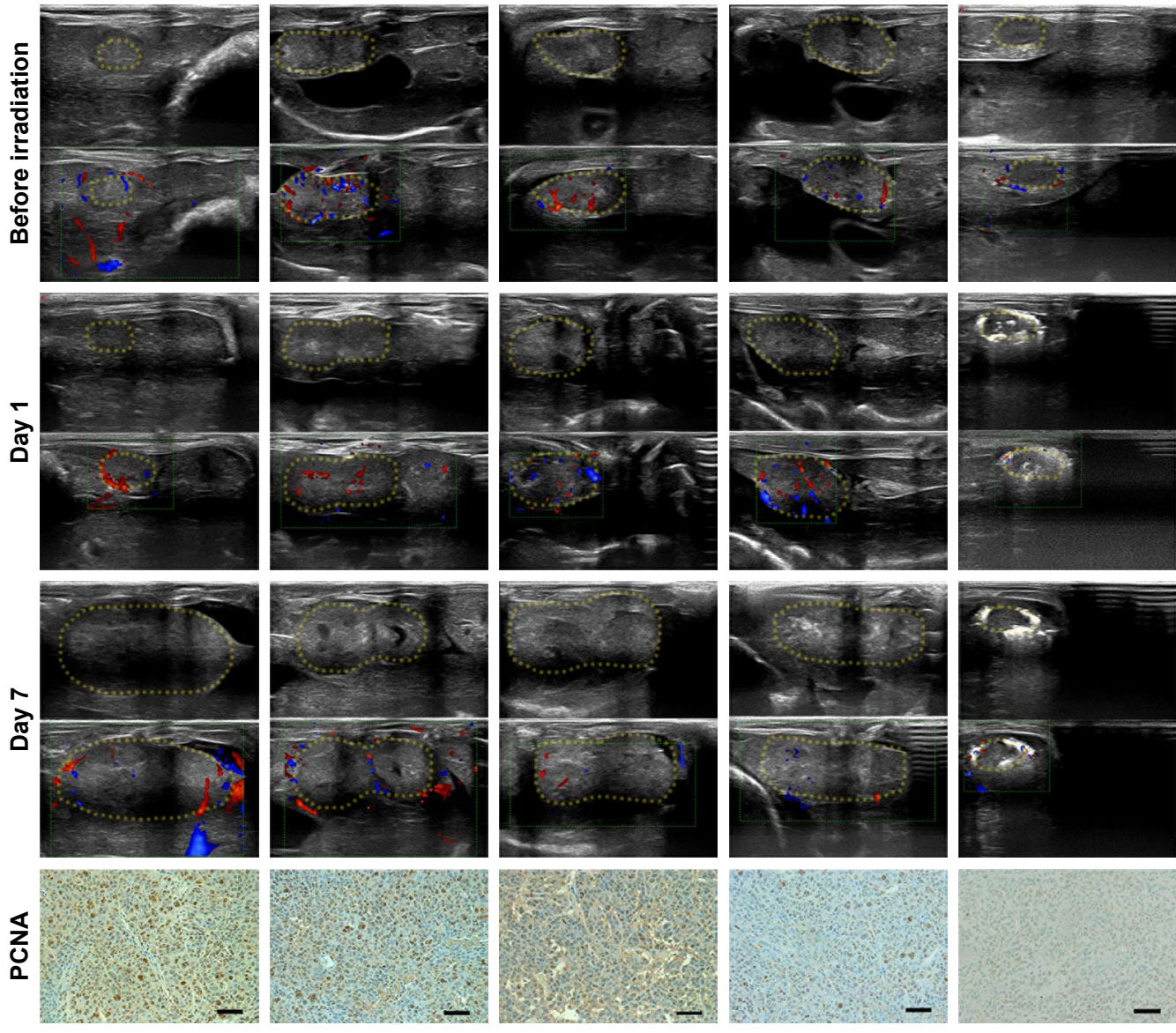

B

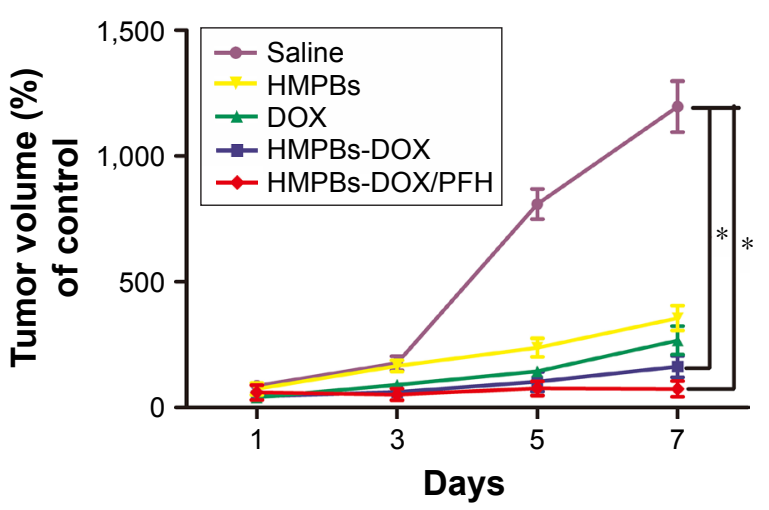

C

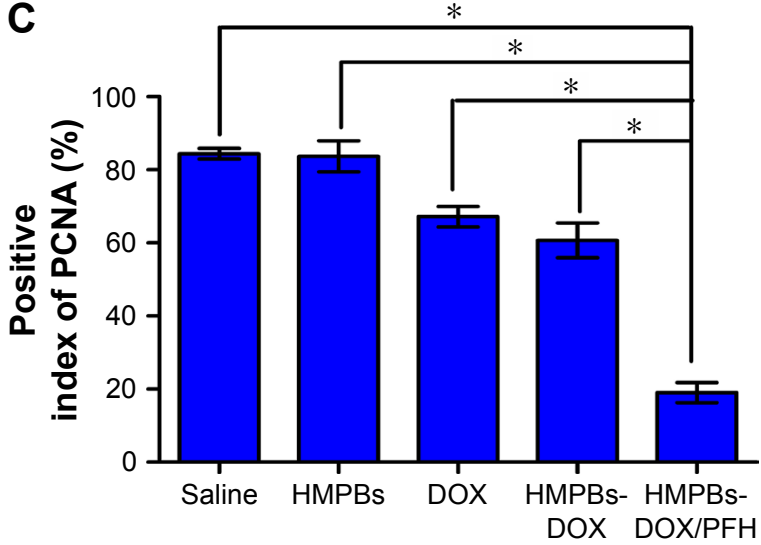

Figure 7 HMPBs-DOX/PFH for the synergistic HIFU liver cancer surgery.

Notes: (A) Ultrasound imaging of VX2 tumor treated with saline and HMPBs-DOX/PFH before and on days I and 7 after HIFU treatment. PCNA photographs of the VX2 tumor on day 7 after therapy. The scale bar in (A) indicates $50 \mu \mathrm{m}$. Magnification $\times 400$. (B) The tumor growth curve of tumor volume in different groups (saline, HMPBs, DOX, HMPBs-DOX, HMPBs-DOX/PFH) after HIFU irradiation $(* p<0.05)$. (C) Positive index of PCNA in different groups after HIFU irradiation $(* p<0.05)$.

Abbreviations: DOX, doxorubicin; HIFU, high-intensity focused ultrasound; HMPBs, hollow mesoporous Prussian blue nanoparticles; PCNA, proliferating cell nuclear antigen; PFH, perfluorohexane; HMPBs-DOX, DOX-encapsulated HMPBs; HMPBs-DOX/PFH, DOX/PFH-encapsulated HMPBs.

efficiency, because vessels around the tumor take the thermal energy away. In this study, an increase in liver tumor size in the control saline group was observed, with an average volume growth rate of $1,196.73 \% \pm 100.82 \%$, with the tumor dimensions determined using ultrasound imaging.
In the HMPBs-DOX/PFH group, the tumor growth rate of $58.13 \% \pm 15.77 \%$ was significantly lower than in other groups $(p<0.05$, Figure 7B). For the evaluation of VX2 tumor cell proliferation in the liver of rabbits after HIFU irradiation, immunohistochemical staining with antibodies 
against PCNA assay was performed (Figure 7C). The positive index of PCNA was $84.42 \% \pm 1.459 \%, 83.6775 \% \pm 4.256 \%$, $67.20 \% \pm 2.798 \%$, and $60.72 \% \pm 4.725 \%$ in the saline, HMPBs, DOX, and HMPBs-DOX groups, respectively. These results indicated that the proliferation of tumors was not affected by HIFU treatment in these four groups. However, the expression of PCNA in the HMPBs-DOX/PFH group was reduced in the necrotic region. The positive index of PCNA was $19.08 \% \pm 2.745 \%(p<0.05)$ compared with the other groups.

\section{Conclusion}

A versatile theranostic agent (HMPBs-DOX/PFH) was developed for achieving highly efficient HIFU/chemotherapy under enhanced imaging guidance. The nanoplatforms have great potential for intensifying ultrasound and MRI dualmodality imaging contrast, which enables better monitoring and evaluation of the process of thermal therapeutics in deep tumors. As a result of the phase transformation performance of PFH, the nanoplatforms also increase the efficacy of HIFU treatment by increasing the ultrasonic cavitation and enhancing the energy deposition. More importantly, by loading anticancer drug DOX into the particle core alongside the PFH liquid, this multifunctional nanoplatform can be selectively vaporized, releasing the drug into the ROI, enabling distinctively synergistic effects of HIFU-enhanced ablation and chemotherapy on any residual tumor tissue, inhibiting possible tumor recurrence, and minimizing systemic side effects. Such a multifunctional nanoplatform, which can integrate dual-modality imaging with improved therapeutic effects, shows great promise in the field of cancer theranostics.

\section{Acknowledgments}

The authors are grateful to Xiang Zhang, Liang Zhang, Hang Zhou, and Danli Sheng for assistance with the ultrasound and MRI and to Qi Wang for technical support with HIFU. This work was financially supported by the National Natural Science Foundation of the People's Republic of China (Grant nos 31630026, 81227801).

\section{Disclosure}

The authors report no conflicts of interest in this work.

\section{References}

1. Simonetti RG, Cammà C, Fiorello F, Politi F, D’Amico G, Pagliaro L. Hepatocellular carcinoma. Dig Dis Sci. 1991;36(7):962-972.

2. Yang JD, Roberts LR. Hepatocellular carcinoma: a global view. Nat Rev Gastroenterol Hepatol. 2010;7(8):448-458.

3. Yamashita T, Ji J, Budhu A, et al. EpCAM-positive hepatocellular carcinoma cells are tumor-initiating cells with stem/progenitor cell features. Gastroenterology. 2009;136(3):1012-1024.
4. Chen X. Advances in non-surgical management of primary liver cancer. World J Gastroenterol. 2014;20(44):16630.

5. Cillo U, Vitale A, Grigoletto F, et al. Prospective validation of the Barcelona clinic liver cancer staging system. J Hepatol. 2006;44(4): 723-731.

6. Sangro B, Carpanese L, Cianni R, et al; European Network on Radioembolization with Yttrium-90 Resin Microspheres (ENRY). Survival after yttrium-90 resin microsphere radioembolization of hepatocellular carcinoma across Barcelona clinic liver cancer stages: a European evaluation. Hepatology. 2011;54(3):868-878.

7. Llovet JM, Di Bisceglie AM, Bruix J, et al; Panel of Experts in HCCDesign Clinical Trials. Design and endpoints of clinical trials in hepatocellular carcinoma. J Natl Cancer Inst. 2008;100(10):698-711.

8. Makuuchi M, Kawasaki S, Noguchi T, et al. Donor hepatectomy for living related partial liver transplantation. Surgery. 1993;113(4):395-402.

9. Makuuchi M, Kosuge T, Takayama T, et al. Surgery for small liver cancers. Semin Surg Oncol. 1993;9(4):298-304.

10. You Y, Wang Z, Ran H, et al. Nanoparticle-enhanced synergistic HIFU ablation and transarterial chemoembolization for efficient cancer therapy. Nanoscale. 2016;8(7):4324-4339.

11. Zhang L, Zhu H, Jin C, et al. High-intensity focused ultrasound (HIFU): effective and safe therapy for hepatocellular carcinoma adjacent to major hepatic veins. Eur Radiol. 2009;19(2):437-445.

12. Kang TW, Rhim H. Recent advances in tumor ablation for hepatocellular carcinoma. Liver Cancer. 2015;4(3):176-187.

13. Zavaglia C, Mancuso A, Foschi A, Rampoldi A. High-intensity focused ultrasound (HIFU) for the treatment of hepatocellular carcinoma: is it time to abandon standard ablative percutaneous treatments? Hepatobiliary Surg Nutr. 2013;2(4):184-187.

14. Ni S, Liu L, Shu Y. Sequential transcatheter arterial chemoembolization, three-dimensional conformal radiotherapy, and high-intensity focused ultrasound treatment for unresectable hepatocellular carcinoma patients. J Biomed Res. 2012;26(4):260-267.

15. Molinari M, Helton S. Hepatic resection versus radiofrequency ablation for hepatocellular carcinoma in cirrhotic individuals not candidates for liver transplantation: a Markov model decision analysis. Am J Surg. 2009;198(3):396-406.

16. Goldberg SN, Solbiati L, Hahn PF, et al. Large-volume tissue ablation with radio frequency by using a clustered, internally cooled electrode technique: laboratory and clinical experience in liver metastases. Radiology. 1998;209(2):371-379.

17. Billard BE, Hynynen K, Roemer RBR. Effects of physical parameters on high temperature ultrasound hyperthermia. Ultrasound Med Biol. 1990;16(4):409-420.

18. Ma M, Xu HX, Chen HR, et al. A drug-perfluorocarbon nanoemulsion with an ultrathin silica coating for the synergistic effect of chemotherapy and ablation by high-intensity focused ultrasound. Adv Mater. 2014; 26(43):7378-7385.

19. Zhang X, Zheng Y, Wang Z, et al. Methotrexate-loaded PLGA nanobubbles for ultrasound imaging and synergistic targeted therapy of residual tumor during HIFU ablation. Biomaterials. 2014;35(19):5148-5161.

20. Zhou Y, Wang Z, Chen Y, et al. Microbubbles from gas-generating perfluorohexane nanoemulsions for targeted temperature-sensitive ultrasonography and synergistic HIFU ablation of tumors. Adv Mater. 2013;25(30):4123-4130.

21. Niu D, Wang X, Li Y, et al. Facile synthesis of magnetite/ perfluorocarbon co-loaded organic/inorganic hybrid vesicles for dual-modality ultrasound/magnetic resonance imaging and imagingguided high-intensity focused ultrasound ablation. Adv Mater. 2013; 25(19):2686-2692.

22. Sun Y, Zheng Y, Ran H, et al. Superparamagnetic PLGA-iron oxide microcapsules for dual-modality US/MR imaging and high intensity focused US breast cancer ablation. Biomaterials. 2012;33(24): 5854-5864.

23. Wang X, Chen H, Chen Y, et al. Perfluorohexane-encapsulated mesoporous silica nanocapsules as enhancement agents for highly efficient high intensity focused ultrasound (HIFU). Adv Mater. 2012;24(6): 785-791. 
24. Zhang M, Fabiilli ML, Haworth KJ, et al. Acoustic droplet vaporization for enhancement of thermal ablation by high intensity focused ultrasound. Acad Radiol. 2011;18(9):1123-1132.

25. Yao MH, Ma M, Chen Y, et al. Multifunctional Bi $\mathrm{S}_{3} / \mathrm{PLGA}$ nanocapsule for combined HIFU/radiation therapy. Biomaterials. 2014; 35(28):8197-8205.

26. Cheng Z, Al Zaki A, Hui JZ, Muzykantov VR, Tsourkas A. Multifunctional nanoparticles: cost versus benefit of adding targeting and imaging capabilities. Science. 2012;338(6109):903-910.

27. Li Y, Lin T, Luo Y, et al. A smart and versatile theranostic nanomedicine platform based on nanoporphyrin. Nat Commun. 2014;5:4712.

28. Fu G, Liu W, Feng S, Yue X. Prussian blue nanoparticles operate as a new generation of photothermal ablation agents for cancer therapy. Chem Commun. 2012;48:11567-11569.

29. Jing L, Liang X, Deng Z, et al. Prussian blue coated gold nanoparticles for simultaneous photoacoustic/CT bimodal imaging and photothermal ablation of cancer. Biomaterials. 2014;35(22):5814-5821.
30. Hu M, Furukawa S, Ohtani R, et al. Synthesis of Prussian blue nanoparticles with a hollow interior by controlled chemical etching. Angew Chemie Int Ed. 2012;51(4):984-988.

31. Ming H, Torad NLK, Chiang Y-D, Wu C, Yamauchi Y, Wu KC-W. Size- and shape-controlled synthesis of Prussian blue nanoparticles by a polyvinylpyrrolidone-assisted crystallization process. CrystEngComm. 2012;14(10):3387

32. Shokouhimehr M, Soehnlen ES, Hao J, et al. Dual purpose Prussian blue nanoparticles for cellular imaging and drug delivery: a new generation of T1-weighted MRI contrast and small molecule delivery agents. J Mater Chem. 2010;20(25):5251.

33. Wu M, Meng Q, Chen Y, et al. Large pore-sized hollow mesoporous organosilica for redox-responsive gene delivery and synergistic cancer chemotherapy. Adv Mater. 2016;28(10):1963-1969. 


\section{Supplementary materials}

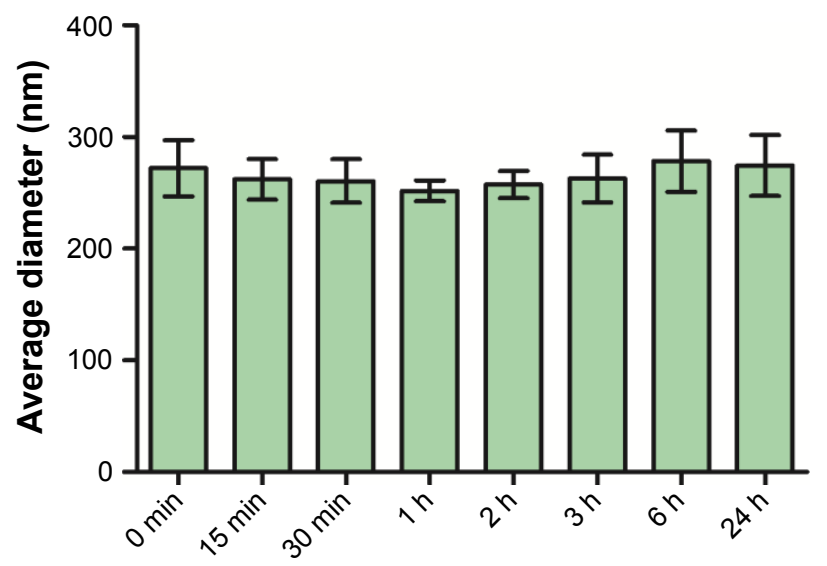

Figure SI Average diameters of HMPBs-DOX/PFH under $37^{\circ} \mathrm{C}$ at different time points.

Note: The average diameter of the HMPBs-DOX/PFH had no obvious change at $37^{\circ} \mathrm{C}$ during $24 \mathrm{~h}$, which demonstrated that the hybrid mesoporous nanoparticles had good stability.

Abbreviations: DOX, doxorubicin; HMPBs, hollow mesoporous Prussian blue nanoparticles; PFH, perfluorohexane.

HMPBs-DOX
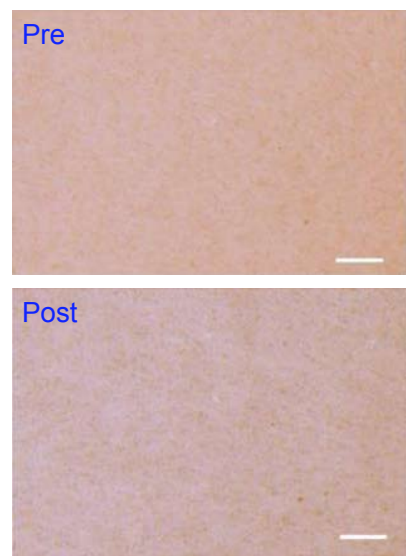

HMPBs-DOX/PFH
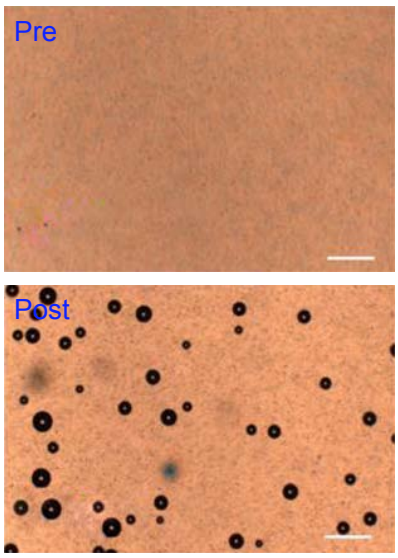

Figure S2 Optical microscopy images of HMPBs-DOX and HMPBs-DOX/PFH before and after heating.

Note: A large number of microbubbles were observed after the heat treatment of HMPBs-DOX/PFH, while the HMPBs-DOX alone at the same concentration did not generate any microbubbles in the heated area.

Abbreviations: DOX, doxorubicin; HMPBs, hollow mesoporous Prussian blue nanoparticles; PFH, perfluorohexane.

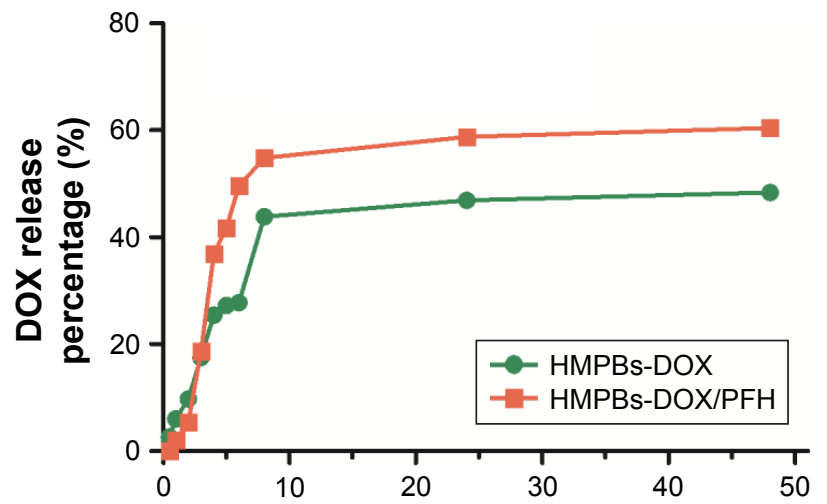

(h)

Figure S3 The DOX release curves in the HMPBs-DOX/PFH and HMPBs-DOX groups with HIFU exposure at $120 \mathrm{~W}$ for $5 \mathrm{~s}$.

Note: The DOX release percentage of HMPBs-DOX/PFH group was $60.4137 \%$, which was higher than the one of HMPBs-DOX group (48.397\%) in $48 \mathrm{~h}$.

Abbreviations: DOX, doxorubicin; HIFU, high-intensity focused ultrasound; HMPBs, hollow mesoporous Prussian blue nanoparticles; PFH, perfluorohexane. 

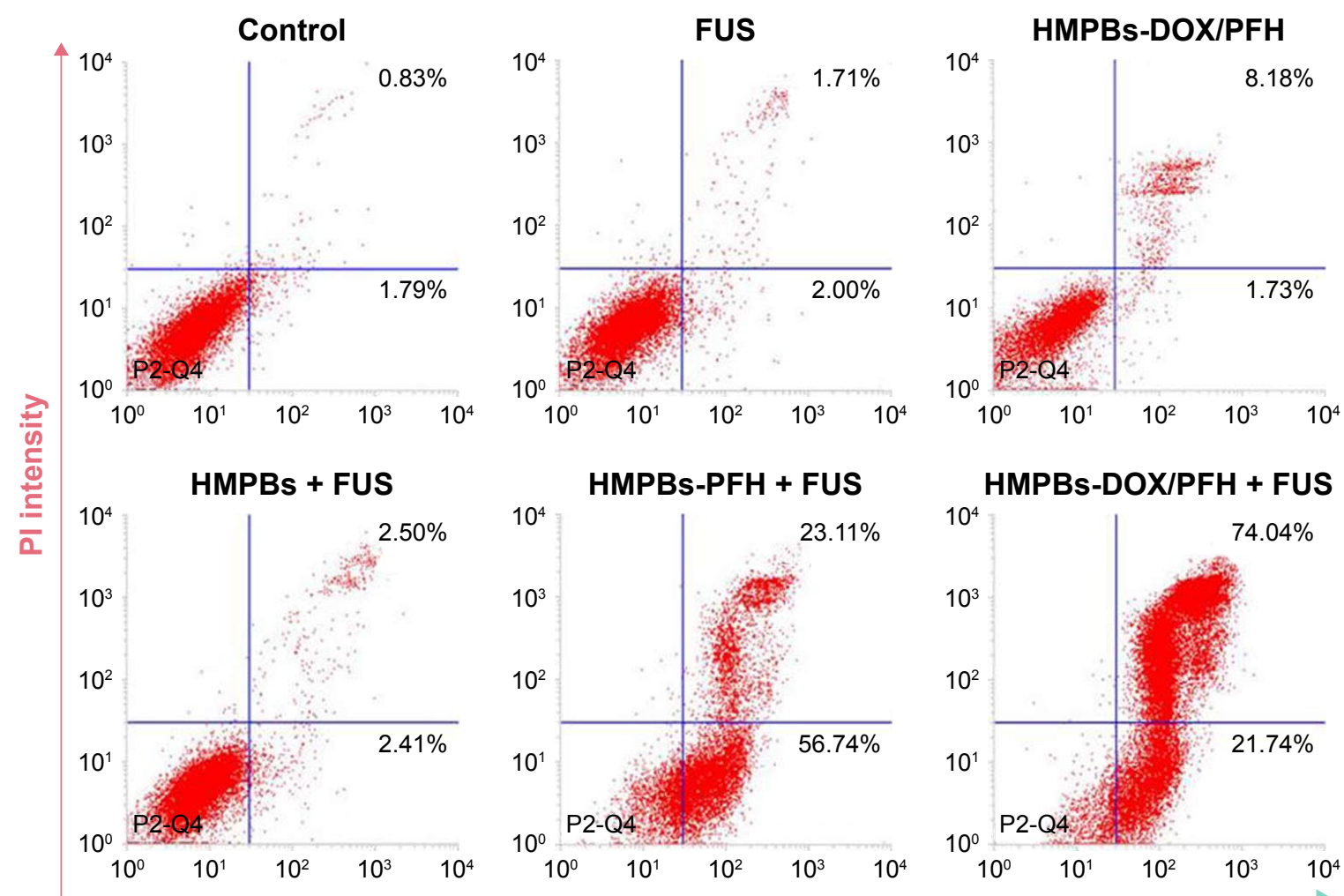

Annexin-V FITC intensity

Figure S4 The quantitative apoptosis of MDA-MB-23I cells with different treatments (control group, FUS alone, HMPBs-DOX/PFH alone, HMPBs + FUS, HMPBs-PFH + FUS, and HMPBs-DOX/PFH + FUS) was detected by flow cytometry.

Notes: The apoptosis efficiencies for the treatment (control group, FUS alone, HMPBs-DOX/PFH alone, HMPBs +FUS, HMPBs-PFH+FUS, and HMPBs-DOX/PFH+FUS) are $2.62 \%, 3.71 \%, 9.91 \%, 4.91 \%, 79.85 \%$, and $95.78 \%$, respectively. It shows that FUS-mediated HMPBs-PFH and HMPBs-DOX/PFH groups experienced the conspicuous cell apoptosis.

Abbreviations: DOX, doxorubicin; FITC, fluorescein isothiocyanate; FUS, focused ultrasound; HMPBs, hollow mesoporous Prussian blue nanoparticles; PFH, perfluorohexane; PI, propidium iodide.

International Journal of Nanomedicine

Dovepress

\section{Publish your work in this journal}

The International Journal of Nanomedicine is an international, peerreviewed journal focusing on the application of nanotechnology in diagnostics, therapeutics, and drug delivery systems throughou the biomedical field. This journal is indexed on PubMed Central, MedLine, CAS, SciSearch ${ }^{\circledR}$, Current Contents ${ }^{\circledR} /$ Clinical Medicine,
Journal Citation Reports/Science Edition, EMBase, Scopus and the Elsevier Bibliographic databases. The manuscript management system is completely online and includes a very quick and fair peer-review system, which is all easy to use. Visit http://www.dovepress.com/ testimonials.php to read real quotes from published authors. 